\title{
Effective field theory of dissipative fluids (II): classical limit, dynamical KMS symmetry and entropy current
}

\author{
Paolo Glorioso, ${ }^{a}$ Michael Crossley ${ }^{b}$ and Hong Liu ${ }^{b}$ \\ ${ }^{a}$ Kadanoff Center for Theoretical Physics and Enrico Fermi Institute, University of Chicago, \\ Chicago, IL 6063\%, U.S.A. \\ ${ }^{b}$ Center for Theoretical Physics, Massachusetts Institute of Technology, \\ Cambridge, MA 02139, U.S.A. \\ E-mail: paolog@uchicago.edu, michael.james.crossley@gmail.com, \\ hong_liu@mit.edu
}

ABSTRACT: In this paper we further develop the fluctuating hydrodynamics proposed in [1] in a number of ways. We first work out in detail the classical limit of the hydrodynamical action, which exhibits many simplifications. In particular, this enables a transparent formulation of the action in physical spacetime in the presence of arbitrary external fields. It also helps to clarify issues related to field redefinitions and frame choices. We then propose that the action is invariant under a $Z_{2}$ symmetry to which we refer as the dynamical KMS symmetry. The dynamical KMS symmetry is physically equivalent to the previously proposed local KMS condition in the classical limit, but is more convenient to implement and more general. It is applicable to any states in local equilibrium rather than just thermal density matrix perturbed by external background fields. Finally we elaborate the formulation for a conformal fluid, which contains some new features, and work out the explicit form of the entropy current to second order in derivatives for a neutral conformal fluid.

Keywords: Effective Field Theories, Space-Time Symmetries, Quantum Dissipative Systems

ARXIV EPRINT: 1701.07817 


\section{Contents}

1 Introduction $\quad 2$

2 Action for fluctuating hydrodynamics $\quad 6$

$\begin{array}{lll}2.1 & \text { Action in the fluid spacetime } & 6\end{array}$

2.2 Formulation in physical spacetime $\quad 8$

$\begin{array}{lll}2.3 & \text { Consequences of local KMS condition } & 9\end{array}$

3 Classical limit $\quad 11$

$\begin{array}{lll}3.1 & \text { Small } \hbar \text { expansion } & 11\end{array}$

$\begin{array}{lll}3.2 & \text { Physical space formulation } & 13\end{array}$

4 Dynamical KMS symmetry $\quad \mathbf{1 5}$

$\begin{array}{llr}4.1 \text { Proposal } & 16\end{array}$

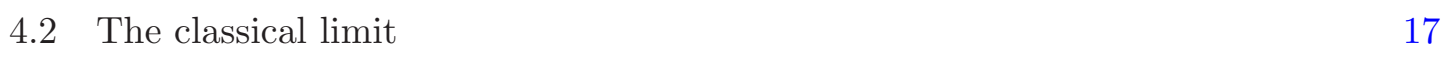

$\begin{array}{lll}4.3 & \text { Open issues at finite } \hbar & 19\end{array}$

5 Dynamical KMS invariance and entropy current $\quad 19$

$\begin{array}{ll}5.1 \text { Dynamical KMS invariance } & 19\end{array}$

5.2 Explicit tensor analysis to first order in derivative expansion 22

$\begin{array}{lll}5.3 & \text { Entropy current } & 23\end{array}$

6 Frame choices from field redefinitions $\quad 24$

6.1 General discussion of field redefinitions 24

6.2 Landau frame and generalized Landau frame 26

6.3 First order action in the generalized Landau frame 27

6.4 Dynamical KMS condition in Landau frame 29

6.5 Entropy current in Landau frame 30

7 Conformal fluids at second order in derivatives 31

$\begin{array}{lll}7.1 & \text { Conformal fluids } & 32\end{array}$

$\begin{array}{ll}7.2 & \text { Analysis at second order } \\ 7.3 & \text { Entropy }\end{array}$

$\begin{array}{lll}7.3 & \text { Entropy current at second order } & 35\end{array}$

8 Conclusions and discussions $\quad 36$

$\begin{array}{ll}\text { A A simple argument } & 37\end{array}$

B Absorbing $V^{\mu}$ by total derivatives $\quad 37$

$\begin{array}{lll}\text { C A special basis } & 38\end{array}$

$\begin{array}{ll}\text { D Action for conformal fluids in fluid spacetime } & 39\end{array}$

$\begin{array}{lll}\text { E Useful identities } & 41\end{array}$ 


\section{Introduction}

For a quantum many-body system in local thermal equilibrium, in generic situations, the only long-lived modes are those associated with conserved quantities such as energymomentum tensor and conserved currents for some global symmetries. Recently, using this as a starting point we proposed a new formulation of fluctuating hydrodynamics as a universal low energy effective theory of a quantum many-body system at a finite temperature [1]. ${ }^{1}$ The theory gives a path integral formulation of hydrodynamics which systematically incorporates effects of fluctuations, including nonlinear interactions involving noises as well as non-equilibrium fluctuation-dissipation relations. The conventional hydrodynamical equations of motion are recovered as saddle point equations, and the stochastic hydrodynamics is recovered by truncating the noise part of the action to quadratic level.

We now summarize the salient aspects of the theory of [1]. For definiteness we will consider a system with a U(1) global symmetry. Consider the closed time path (CTP) generating functional for the stress tensor and $\mathrm{U}(1)$ current in a thermal density matrix $\rho_{0}$

$$
\begin{aligned}
e^{W\left[g_{1 \mu \nu}, A_{1 \mu} ; g_{2 \mu \nu}, A_{2 \mu}\right]} & \equiv \operatorname{Tr}\left[U\left(+\infty,-\infty ; g_{1 \mu \nu}, A_{1 \mu}\right) \rho_{0} U^{\dagger}\left(+\infty,-\infty ; g_{2 \mu \nu}, A_{2 \mu}\right)\right] \\
& =\int D \chi_{1} D \chi_{2} e^{i I_{\text {hydro }}\left[\chi_{1}, g_{1}, A_{1} ; \chi_{2}, g_{2}, A_{2}\right]}
\end{aligned}
$$

where $U\left(t_{2}, t_{1} ; g_{\mu \nu}, A_{\mu}\right)$ is the evolution operator of the system from $t_{1}$ to $t_{2}$ in the presence of a spacetime metric $g_{\mu \nu}$ (sources for stress tensor) and an external vector field $A_{\mu}$ (sources for the $\mathrm{U}(1)$ current). The sources are taken to be slowly varying functions and there are two copies of them, one for each leg of the CTP contour. The second line (1.2) should be imagined as obtained by integrating out all the fast modes of the system with $\chi_{1,2}$ denoting the remaining slow modes (hydrodynamical modes), and $I_{\text {hydro }}$ is the effective action for them. Again there are two copies of hydrodynamical modes. While in practice the integrations from (1.1) to (1.2) cannot be done, $I_{\text {hydro }}$ can be obtained as the most general local action once we have identified the dynamical variables $\chi_{1,2}$ and the symmetries $I_{\text {hydro }}$ should obey.

In [1] we developed an "integrating-in" procedure to identify the slow degrees of freedom associated with a conserved quantity. For the stress tensor this leads to a doubled version of the Lagrange description of fluid flows, with the corresponding $\chi_{1,2}$ given by mappings $X_{1,2}^{\mu}\left(\sigma^{a}\right)$ between a "fluid spacetime," whose coordinates $\sigma^{a}$ label fluid elements and their internal clocks, and the two copies of physical spacetimes with coordinates $X_{1,2}^{\mu}$ respectively. See figure 1. The slow degrees of freedom for the U(1) current are $\varphi_{1,2}\left(\sigma^{a}\right)$ which can be interpreted as $\mathrm{U}(1)$ phase rotations in two physical spacetimes associated for each fluid elements. ${ }^{2}$ One also needs to introduce an additional scalar field $\tau\left(\sigma^{a}\right)$ which gives the local proper temperature in fluid spacetime

$$
T(\sigma)=\frac{1}{\beta(\sigma)}=T_{0} e^{-\tau(\sigma)} .
$$

\footnotetext{
${ }^{1}$ For other recent discussions of fluctuating hydrodynamics, see $[2-16,16,17,17-21]$ and in holographic context [22-24].

${ }^{2}$ Other recent work which uses effective action from CTP to describe fluctuating hydrodynamics include [11, 14-17]. In particular similar variables were also used in [11-17].
} 
(a)

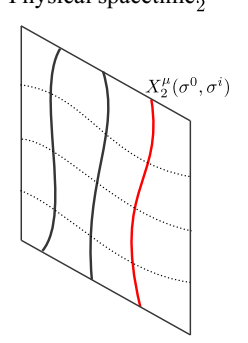

Fluid spacetime

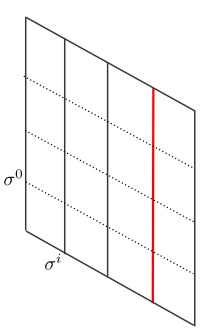

Physical spacetime $_{1}$

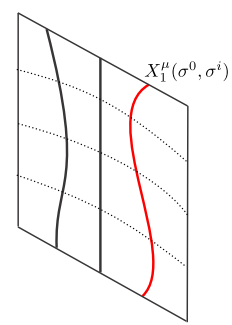

Figure 1. Relations between the fluid spacetime and two copies of physical spacetimes. The red straight line in the fluid spacetime with constant $\sigma^{i}$ is mapped by $X_{1,2}^{\mu}\left(\sigma^{0}, \sigma^{i}\right)$ to physical spacetime trajectories (also in red) of the corresponding fluid element.

$T_{0}=\frac{1}{\beta_{0}}$ is the temperature at infinities where we take all external sources and dynamical fields to vanish. Note that there is only one $\tau$ field rather than two copies. The standard variables such as local velocity and chemical potential are built from symmetric combinations of $X_{1,2}^{\mu}, \varphi_{1,2}$, while their antisymmetric combinations can be interpreted as corresponding "noises."

It turns out these variables are not enough. In order to ensure the unitarity of (1.1) one needs to introduce anti-commuting partners ("ghosts") for dynamical variables and require the full action to satisfy a BRST-type fermionic symmetry. ${ }^{3}$ In this paper we will focus on the bosonic part and so will suppress all ghost variables below. The structure with ghost sector is elucidated in [26, 27].

In terms of variables described above (1.2) can be written more explicitly as

$$
e^{W\left[g_{1}, A_{1} ; g_{2}, A_{2}\right]}=\int D X_{1} D X_{2} D \varphi_{1} D \varphi_{2} D \tau e^{i I_{\mathrm{hydro}}\left[h_{1}, B_{1} ; h_{2}, B_{2} ; \tau\right]},
$$

where $(s=1,2$ and no summation over $s)$

$$
h_{s a b}(\sigma)=\frac{\partial X_{s}^{\mu}}{\partial \sigma^{a}} g_{s \mu \nu}\left(X_{s}(\sigma)\right) \frac{\partial X_{s}^{\nu}}{\partial \sigma^{b}}, \quad B_{s a}(\sigma)=\frac{\partial X_{s}^{\mu}}{\partial \sigma^{a}} A_{s \mu}\left(X_{s}(\sigma)\right)+\partial_{a} \varphi_{s}(\sigma) .
$$

$h_{1,2}$ are pull-backs of the spacetime metrics to the fluid spacetime and similarly the first terms in $B_{1,2}$ are pull-backs of spacetime vector sources. Due to conservation of the stress tensor and $\mathrm{U}(1)$ current, $W\left[g_{1}, A_{1} ; g_{2}, A_{2}\right]$ should be invariant under independent diffeomorphisms of the two copies of spacetime and independent gauge transformations of $A_{1}, A_{2}$. This is ensured by $I_{\text {hydro }}$ being a local action of $h_{1,2}, \tau, B_{1,2}$. By construction $h_{1,2}$ and $B_{1,2}$ are invariant under $(s=1,2)$

$$
\begin{aligned}
g_{s \mu \nu}^{\prime}\left(X_{s}^{\prime}\right) & =\frac{\partial X_{s}^{\lambda}}{\partial X_{s}^{\prime \mu}} \frac{\partial X_{s}^{\rho}}{\partial X_{s}^{\prime \nu}} g_{s \lambda \rho}\left(X_{s}\right), & A_{s \mu}^{\prime}\left(X_{s}^{\prime}\right) & =\frac{\partial X_{s}^{\lambda}}{\partial X_{s}^{\prime \mu}} A_{s \lambda}\left(X_{s}\right), \quad X_{s}^{\prime \mu}(\sigma)=f_{s}^{\mu}\left(X_{s}(\sigma)\right) \\
A_{s \mu}^{\prime} & =A_{s \mu}-\partial_{\mu} \lambda_{s}\left(X_{s}\right), & \varphi_{s}^{\prime}(\sigma) & =\varphi_{s}(\sigma)+\lambda_{s}\left(X_{s}(\sigma)\right)
\end{aligned}
$$

for arbitrary functions $f_{1}, f_{2}$ and $\lambda_{1}, \lambda_{2}$.

\footnotetext{
${ }^{3}$ The need for anti-commuting degrees of freedom and BRST symmetry in path integral formulation of stochastic systems has been well known since 1970's. See e.g. [25] for a review. Their presence for fluctuating hydrodynamics has also been discussed recently in [16-19].
} 
$I_{\text {hydro }}$ further satisfies the following symmetry conditions:

1. Invariant under spatial and time diffeomorphisms in the fluid spacetime

$$
\begin{aligned}
\sigma^{i} & \rightarrow \sigma^{\prime i}\left(\sigma^{i}\right), & \sigma^{0} & \rightarrow \sigma^{0} \\
\sigma^{0} & \rightarrow \sigma^{\prime 0}=f\left(\sigma^{0}, \sigma^{i}\right), & \sigma^{i} & \rightarrow \sigma^{i} .
\end{aligned}
$$

These symmetry conditions define a fluid.

2. Invariant under a diagonal spatial-dependent shift

$$
\varphi_{r} \rightarrow \varphi_{r}-\lambda\left(\sigma^{i}\right), \quad \varphi_{a} \rightarrow \varphi_{a}
$$

with $\varphi_{r}=\frac{1}{2}\left(\varphi_{1}+\varphi_{2}\right)$ and $\varphi_{a}=\varphi_{1}-\varphi_{2}$. This condition specifies a normal fluid as compared to a superfluid.

3. Invariant under a $Z_{2}$ reflection symmetry

$$
I_{\text {hydro }}^{*}\left[h_{1}, B_{1} ; h_{2}, B_{2} ; \tau\right]=-I_{\text {hydro }}\left[h_{2}, B_{2} ; h_{1}, B_{1} ; \tau\right]
$$

which is needed to be consistent with the behavior of (1.1) under complex conjugation. The condition implies that $I_{\text {hydro }}$ must be complex and in particular the imaginary part of the action must be even under exchange of 1,2 indices. For the path integral (1.4) to be bounded we further require that

$$
\operatorname{Im} I_{\text {hydro }} \geq 0
$$

for any dynamical variables and external sources.

4. Vanish when we set all the sources and dynamical fields of the two legs to be equal, i.e.

$$
I[h, B ; h, B ; \tau]=0 .
$$

Equations (1.11)-(1.13) are all consequences of unitary time evolution.

5. Local KMS condition which can be stated as follows. Setting the dynamical fields to "background" values

$$
X_{1,2}^{\mu}=\sigma^{a} \delta_{a}^{\mu}, \quad \varphi_{1,2}=0, \quad e^{\tau}=\sqrt{-g_{r 00}}, \quad g_{r 00}=\frac{1}{2}\left(g_{100}+g_{200}\right) .
$$

in the action and denoting the resulting expression as $I_{s}\left[g_{1}, A_{1} ; g_{2}, A_{2}\right]$, we then impose that

$$
I_{s}\left[g_{1}, g_{2}, A_{1}, A_{2}\right]=I_{s}\left[\tilde{g}_{1}, \tilde{g}_{2}, \tilde{A}_{1}, \tilde{A}_{2}\right]
$$

where the tilde variables are defined as

$$
\begin{array}{llrl}
\tilde{g}_{1 \mu \nu}(x) & =g_{1 \mu \nu}(-t+i \theta,-\vec{x}), & \tilde{A}_{1 \mu}(x)=A_{1 \mu}(-t+i \theta,-\vec{x}), \\
\tilde{g}_{2 \mu \nu}(x)=g_{2 \mu \nu}\left(-t-i\left(\beta_{0}-\theta\right),-\vec{x}\right), & \tilde{A}_{2 \mu}(x)=A_{2 \mu}\left(-t-i\left(\beta_{0}-\theta\right),-\vec{x}\right) .
\end{array}
$$


for arbitrary $\theta \in\left[0, \beta_{0}\right]$. This condition is to ensure that for $\rho_{0}$ given by the thermal density matrix (1.1) satisfies a condition ${ }^{4}$ obtained by combining the Kubo-MartinSchwinger (KMS) condition with $\mathcal{P} \mathcal{T}$

$$
W\left[g_{1}(x), A_{1}(x) ; g_{2}(x), A_{2}(x)\right]=W\left[\tilde{g}_{1}(x), \tilde{A}_{1}(x) ; \tilde{g}_{2}(x), \tilde{A}_{2}(x)\right] .
$$

The action $I_{\text {hydro }}\left[h_{2}, B_{2} ; h_{1}, B_{1} ; \tau\right]$ is then obtained as the most general local action consistent with the above conditions. In particular, at the level of equations of motion the local KMS condition recovers all the standard constraints of hydrodynamics from the entropy current condition and linear Onsager relations. Furthermore, it leads to new constraints from nonlinear generalizations of the Onsager relations, and non-equilibrium fluctuationdissipation relations.

In this paper we further develop the formulation in a number of ways:

1. We work out in detail the classical limit of $I_{\text {hydro }}$. There are many simplifications in this limit. In particular, this enables a transparent formulation of the fluctuating hydrodynamics in physical spacetime in the presence of arbitrary external fields. It also helps to clarify issues related to field redefinitions and frame choice.

2. We replace the local KMS condition by an alternative formulation, which directly acts on the dynamical fields. To distinguish it from (1.15) we will refer to it as dynamical KMS symmetry (or condition). The dynamical formulation is equivalent to the previous one in the classical limit, but is more convenient to implement and more general. It should be applicable to any states $\rho_{0}$ in local equilibrium rather than just thermal density matrix perturbed by external background fields. The dynamical formulation has been recently used in [28] to prove the local second law of thermodynamics and also leads to an explicit construction of the entropy current from a Noether-like procedure.

3. We elaborate the formulation for a conformal fluid, which contains some new features. We also give the explicit action of a neutral conformal fluid to the second order in derivatives and work out the corresponding entropy current following the general construction presented in [28].

The plan of the paper is as follows. In next section to help set up the notations we review the action of [1] as well as implications of the local KMS condition. In section 3 we discuss the classical limit. In section 4 we present the formulation of dynamical KMS symmetry. In section 5 we discuss the implications of dynamical KMS symmetry on the action in the classical limit and work out the entropy current to first order in derivatives. In section 6 we discuss field redefinitions and frame choices. In section 7 we discuss formulation of fluctuating hydrodynamics for a conformal fluid, and work out the action and the entropy

\footnotetext{
${ }^{4}$ As explained in detail in [1], KMS condition relates $W$ to a time-reversed one. To obtain a condition on $W$ itself one needs to combine it with a time reversal symmetry. Depending on the situation one could combine it with $\mathcal{T}$ or $\mathcal{P} \mathcal{T}$ or $\mathcal{C P} \mathcal{T}$. For our discussion of effective theory for a charged fluid we choose $\mathcal{P} \mathcal{T}$ for definiteness.
} 
current for a neutral conformal fluid to second order in derivatives. We conclude with a brief discussion of the quantum regime in section 8 .

\section{Action for fluctuating hydrodynamics}

In this section to set up the notations we review the action for fluctuating hydrodynamics for a relativistic system with a $\mathrm{U}(1)$ global symmetry. We will only be concerned with the bosonic action.

\subsection{Action in the fluid spacetime}

To implement separate spatial and time diffeomorphisms (1.8)-(1.9), it is convenient to decompose $h_{1,2}$ and $B_{1,2}$ into objects which have good transformation properties under them (below $s=1,2$ )

$$
\begin{aligned}
h_{s a b} d \sigma^{a} d \sigma^{b} & =-b_{s}^{2}\left(d \sigma^{0}-v_{s i} d \sigma^{i}\right)^{2}+a_{s i j} d \sigma^{i} d \sigma^{j}, \\
B_{s a} d \sigma^{a} & =\mu_{s} b\left(d \sigma^{0}-v_{s i} d \sigma^{i}\right)+\mathfrak{b}_{s i} d \sigma^{i} .
\end{aligned}
$$

To implement (1.11)-(1.13) it is convenient to further introduce symmetric and antisymmetric combinations $(r-a$ variables)

$$
\begin{aligned}
& E_{r}=\frac{1}{2}\left(b_{1}+b_{2}\right), \quad E_{a}=\log \left(b_{2}^{-1} b_{1}\right), \quad V_{a i}=E_{r}\left(v_{1 i}-v_{2 i}\right), \quad V_{r i}=\frac{1}{2} E_{r}\left(v_{1 i}+v_{2 i}\right), \\
& a_{r i j}=\frac{1}{2}\left(a_{1 i j}+a_{2 i j}\right), \quad \chi_{a}=\frac{1}{2} \log \operatorname{det}\left(a_{2}^{-1} a_{1}\right), \quad \Xi=\log \left(\hat{a}_{2}^{-1} \hat{a}_{1}\right), \\
& \mu_{r}=\frac{1}{2}\left(\mu_{1}+\mu_{2}\right), \quad \mu_{a}=\mu_{1}-\mu_{2}, \quad \mathfrak{b}_{r i}=\frac{1}{2}\left(\mathfrak{b}_{1 i}+\mathfrak{b}_{2 i}\right), \quad \mathfrak{b}_{a i}=\mathfrak{b}_{1 i}-\mathfrak{b}_{2 i}
\end{aligned}
$$

where $\hat{a}_{1,2}$ denotes the unit determinant part of $a_{1,2}$ and thus $\Xi$ is traceless. Note that $\tau$ should be considered as a $r$-variable. $\mu_{r}$ can be identified as the local chemical potential.

The most general action which satisfies the symmetries listed in the Introduction can be readily constructed using (2.3)-(2.5) and their derivatives

$$
I_{\text {hydro }}=\int d^{d} \sigma \sqrt{a_{r}} E_{r} \mathcal{L}
$$

where $\mathcal{L}$ is a scalar under (1.8)-(1.9), and can be written as a double expansion in terms of the number of $a$-type fields (i.e. expanding in noises), and the number of derivatives. More explicitly,

$$
I_{\text {hydro }}=I^{(1)}+I^{(2)}+\cdots, \quad \mathcal{L}=\mathcal{L}^{(1)}+\mathcal{L}^{(2)}+\cdots,
$$

where $\mathcal{L}^{(m)}$ contains $m$ factors of $a$-fields. From $(1.11), \mathcal{L}^{(m)}$ is pure imaginary for even $m$ and real for odd $m$. Each $\mathcal{L}^{(m)}$ can be further expanded in the number of derivatives

$$
\mathcal{L}^{(n)}=\mathcal{L}^{(n, 0)}+\mathcal{L}^{(n, 1)}+\cdots
$$


with $\mathcal{L}^{(n, m)}$ containing $m$ derivatives. To first order in derivatives the most general $\mathcal{L}^{(1,1)}$ can be written as $^{5}$

$$
\begin{aligned}
\mathcal{L}^{(1,1)}= & -f_{1} E_{a}+f_{2} \chi_{a}+f_{3} \nu_{a}-\frac{\eta}{2} \Xi^{i j} D_{0} a_{r i j}-\lambda_{1} V_{a}^{i} D_{i} E_{r}-\lambda_{2} \mathfrak{c}_{a}^{i} \hat{D}_{0} \mathfrak{b}_{r i}+\lambda_{12} V_{a}^{i} \hat{D}_{0} \mathfrak{b}_{r i} \\
& +\lambda_{21} \mathfrak{c}_{a}^{i} D_{i} E_{r}+\lambda_{5} D_{i} \tau V_{a}^{i}+\lambda_{6} D_{i} \mu_{r} V_{a}^{i}+\lambda_{7} D_{i} \tau \mathfrak{c}_{a}^{i}+\lambda_{8} D_{i} \mu_{r} \mathfrak{c}_{a}^{i}+\cdots
\end{aligned}
$$

where $f_{1,2,3}$ can be further expanded in derivatives as

$$
\begin{aligned}
& f_{1}=\epsilon_{0}+f_{11} D_{0} \tau+f_{12} D_{0}\left(\log \sqrt{\operatorname{det} a_{r}}\right)+f_{13} \beta^{-1}(\sigma) D_{0} \hat{\mu}+\text { higher derivatives, } \\
& f_{2}=p_{0}+f_{21} D_{0} \tau-f_{22} D_{0}\left(\log \sqrt{\operatorname{det} a_{r}}\right)+f_{23} \beta^{-1}(\sigma) D_{0} \hat{\mu}+\text { higher derivatives, } \\
& f_{3}=n_{0}+f_{31} D_{0} \tau+f_{32} D_{0}\left(\log \sqrt{\operatorname{det} a_{r}}\right)-f_{33} \beta^{-1}(\sigma) D_{0} \hat{\mu}+\text { higher derivatives }
\end{aligned}
$$

In the above expressions indices are raised and lowered using $a_{r}$, all coefficients are all real functions of $\mu_{r}$ and $\beta(\sigma)$, and ${ }^{6}$

$$
\nu_{a}=\mu_{a}+E_{a} \mu_{r}, \quad \hat{\mu}=\mu_{r} \beta(\sigma), \quad \mathfrak{c}_{a i}=\mathfrak{b}_{a i}-\mu_{r} V_{a i}, \quad \hat{D}_{0} \mathfrak{b}_{r i} \equiv D_{0} \mathfrak{b}_{r i}-\mu_{r} D_{i} E_{r} .
$$

In (2.9)-(2.13) we have also used various covariant derivatives. For a scalar $\phi$ under time diffeomorphism (1.9), the covariant time derivative is defined as

$$
D_{0} \phi \equiv \frac{1}{E_{r}} \partial_{0} \phi
$$

Note that $E_{r}$ and $V_{r i}$ do not transform as a scalar under (1.9). For them one can define a combined object

$$
D_{i} E_{r} \equiv \frac{1}{E_{r}}\left(\partial_{i} E_{r}+\partial_{0} V_{r i}\right)
$$

which transforms under (1.9) as a scalar and under (1.8) as a vector. The spatial covariant derivatives for a scalar $\phi$ and a vector $\phi_{i}$ under (1.8) are defined respectively as

$$
\begin{aligned}
D_{i} \phi & =\partial_{i} \phi+v_{r i} \partial_{0} \phi \equiv d_{i} \phi, \\
D_{i} \phi_{j} & =d_{i} \phi_{j}-\tilde{\Gamma}_{i j}^{k} \phi_{k},
\end{aligned}
$$

where $d_{i} \equiv \partial_{i}+v_{r i} \partial_{0}$ and

$$
\tilde{\Gamma}_{j k}^{i} \equiv \frac{1}{2} a_{r}^{i l}\left(d_{j} a_{r k l}+d_{k} a_{r j l}-d_{l} a_{r j k}\right)=\Gamma_{j k}^{i}+\frac{1}{2} a_{r}^{i l}\left(v_{r j} \partial_{0} a_{r k l}+v_{r k} \partial_{0} a_{r j l}-v_{r l} \partial_{0} a_{r j k}\right)
$$

with $\Gamma_{j k}^{i}$ the Christoffel symbol corresponding to $a_{r i j}$.

To zeroth order in derivatives the most general $\mathcal{L}^{(2,0)}$ can be written as

$$
\begin{aligned}
-i \mathcal{L}^{(2,0)}= & s_{11} E_{a}^{2}+s_{22} \chi_{a}^{2}+s_{33} \nu_{a}^{2}+2 s_{12} E_{a} \chi_{a}+2 s_{13} E_{a} \nu_{a} \\
& +2 s_{23} \chi_{a} \nu_{a}+r \operatorname{tr} \Xi^{2}+r_{11} V_{a}^{i} V_{a i}+2 r_{12} V_{a}^{i} \mathfrak{c}_{a i}+r_{22} \mathfrak{c}_{a}^{i} \mathfrak{c}_{a i},
\end{aligned}
$$

\footnotetext{
${ }^{5}$ This is the most general form which is valid for all dimensions. For specific dimensions one may introduce more terms using $\epsilon$-symbols.

${ }^{6}$ The choice of these combinations makes the coefficients of various terms in the expressions of the stress tensor and current simpler.
} 
where again all coefficients are real and are functions of $\mu_{r}, \beta(\sigma)$. Given that $\mathcal{L}^{(2)}$ is pure imaginary, in order for the path integral (1.4) to be well defined, the coefficients of (2.19) must be such that the expression is non-negative for any choices of dynamical variables.

In (2.9) and (2.19) we have not imposed (1.14)-(1.15) whose consequences will be discussed separately in section 2.3 .

\subsection{Formulation in physical spacetime}

The action (2.9), (2.19) is formulated in the fluid spacetime. The advantage of this formulation is that the action can be easily coupled to external sources and the symmetries of the theory are easy to implement. A shortcoming is that connections with the dynamics in physical spacetime and the standard form of hydrodynamic equations are not manifest. Alternatively one can formulate the effective action in the physical spacetime. For this purpose, introduce

$$
X_{a}^{\mu}=X_{1}^{\mu}(\sigma)-X_{2}^{\mu}(\sigma), \quad X^{\mu}(\sigma)=\frac{1}{2}\left(X_{1}^{\mu}(\sigma)+X_{2}^{\mu}(\sigma)\right) .
$$

We interpret $X^{\mu}(\sigma)$ as the motion of a fluid element in the physical spacetime (now only one copy) and $X_{a}^{\mu}$ as statistical and quantum noises of that motion. One can then invert $X^{\mu}\left(\sigma^{a}\right)$ to obtain $\sigma^{a}\left(X^{\mu}\right)$, and treat $X^{\mu}$ as the coordinates of the physical spacetime and $\sigma^{a}(X)$ as dynamical variables. Other dynamical variables $X_{a}^{\mu}(\sigma), \varphi_{r, a}(\sigma), \tau(\sigma)$ are now all considered as functions of $X^{\mu}$ through $\sigma^{a}(X)$. To emphasize that $X^{\mu}$ are now simply coordinates and not dynamical variables we will denote them as $x^{\mu}$. The dynamical variables are now $\sigma^{a}(x), X_{a}^{\mu}(x), \varphi_{r, a}(x), \tau(x)$.

The background fields then have the form

$$
g_{1 \mu \nu}\left(x+\frac{1}{2} X^{a}(x)\right), \quad A_{1 \mu}\left(x+\frac{1}{2} X^{a}(x)\right), \quad g_{2 \mu \nu}\left(x-\frac{1}{2} X^{a}(x)\right), \quad A_{2 \mu}\left(x-\frac{1}{2} X^{a}(x)\right)
$$

whose arguments depend on dynamical variables $X_{a}^{\mu}$, and thus should be expanded in $X_{a}^{\mu}$ when performing the noise expansion (2.7) in the action. Direct formulation in physical spacetime is complicated as one does not have a canonical definition of the spacetime metric. The obvious candidate $g=\frac{1}{2}\left(g_{1}+g_{2}\right)$ does not make sense as $g_{1}$ and $g_{2}$ transform under independent diffeomorphisms. Thus one cannot just add them. Similar statement applies to $A=\frac{1}{2}\left(A_{1}+A_{2}\right){ }^{7}$ Nevertheless, one could construct the theory by inverting the action in the fluid spacetime. For example, in the absence of background fields one finds (2.9) can be simply written as

$$
I^{(1)}=\int d^{d} x\left[T^{\mu \nu} \partial_{\mu} X_{a \nu}+J^{\mu} \partial_{\mu} \varphi_{a}\right]+O\left(a^{3}\right)
$$

where $T^{\mu \nu}$ and $J^{\mu}$ are the hydrodynamic stress tensor and U(1) current obtained from (2.9).

\footnotetext{
${ }^{7}$ In next section we will see these difficulties go away in the classical limit.
} 


\subsection{Consequences of local KMS condition}

Now let us turn to the local KMS condition (1.14)-(1.15). We will mention its consequences and then discuss some open issues. Applying to (2.9) and (2.19), we can group consequences of (1.15) into three types (with the first equation of (1.14), $\sigma^{a}=\delta_{\mu}^{a} x^{\mu}$ where $x^{\mu}=\left(x^{0}, \vec{x}\right)$ denotes the spacetime coordinates):

1. For time-independent $g_{1,2}$ and $A_{1,2}, I^{(1)}$ should have a factorized form to leading order $g_{a}=g_{1}-g_{2}$ and $A_{a}=A_{1}-A_{2}$, i.e.

$$
I_{s}^{(1)}=\tilde{W}\left[g_{1}, A_{1}\right]-\tilde{W}\left[g_{2}, A_{2}\right]+O\left(g_{a}^{3}, A_{a}^{3}\right)
$$

where $\tilde{W}[g(\vec{x}), A(\vec{x})]$ is some local functional defined on the spatial part (with coordinate $\vec{x}$ ) of the spacetime and satisfies

$$
\tilde{W}[g(\vec{x}), A(\vec{x})]=\tilde{W}[g(-\vec{x}), A(-\vec{x})] .
$$

Applying (2.23) to (2.9)-(2.12) we find various coefficients in $\mathcal{L}^{(1)}$ should satisfy standard thermodynamic relations

$$
\epsilon_{0}+p_{0}-\mu n_{0}=-\frac{\partial p_{0}}{\partial \tau}, \quad n_{0}=\frac{\partial p_{0}}{\partial \mu},
$$

or

$$
\epsilon_{0}+p_{0}=-\left(\frac{\partial p_{0}}{\partial \tau}\right)_{\hat{\mu}}, \quad n_{0}=\beta \frac{\partial p_{0}}{\partial \hat{\mu}},
$$

and

$$
\lambda_{5}=\lambda_{1}+\mu \lambda_{12}, \quad \lambda_{7}=-\lambda_{21}-\mu \lambda_{2}, \quad \lambda_{6}=\lambda_{8}=0 .
$$

Equations (2.27) reproduce the equality-type constraints from the entropy current. Note that $\tilde{W}$ may be interpreted as the partition function of the system on a stationary manifold with metric $g_{\mu \nu}(\vec{x})$ and external source $A_{\mu}(\vec{x})$, and the above discussion derives the partition function prescription proposed in $[29,30]$.

2. Non-equilibrium Onsager relations

$$
\mathcal{G}_{i j}\left(x, y ; \phi_{i}(\vec{x})\right]=\mathcal{G}_{j i}\left(-y,-x ; \phi_{i}(-\vec{x})\right],
$$

where $\phi_{i}$ collectively denotes $g_{\mu \nu}, A_{\mu}$ with $i$ labeling different components of both $g$ and $A$. $\mathcal{G}_{i j}$ is defined as

$$
\mathcal{G}_{i j}\left(x, y ; \phi_{i}(\vec{x})\right]=\left.\frac{\delta^{2} I_{s}^{(1)}}{\delta \phi_{a i}(x) \phi_{r j}(y)}\right|_{S}
$$

where the subscript $S$ in (2.29) denotes the procedure that after taking the differentiation one should set $g_{1 \mu \nu}=g_{2 \mu \nu}=g_{\mu \nu}, A_{1 \mu}=A_{2 \mu}=A_{\mu}$ with both $g_{\mu \nu}$ and $A_{\mu}$ time-independent. The notation $\mathcal{G}(\cdots]$ highlights that it is a function of $x^{\mu}, y^{\mu}$, but a functional of $\phi_{i}(\vec{x})$. Applying $(2.28)$ to $I^{(1)}$ one finds that

$$
\lambda_{12}=\lambda_{21}, \quad-f_{13}=f_{31}, \quad f_{23}=f_{32}, \quad-f_{12}=f_{21} .
$$


Note that (2.30) are the standard constraints from linear Onsager relations. To the first derivative order of (2.9) there is no difference whether one imposes (2.28) on the full nonlinear action (2.9) or the linearized version (around the equilibrium). But one expects additional nonlinear constraints to start appearing at second derivative order [1].

3. Non-equilibrium fluctuation-dissipation relations which relate parameters of $\mathcal{L}^{(1)}$ and $\mathcal{L}^{(2)}$ :

$$
\begin{aligned}
r & =\frac{\eta}{2} T(\sigma), & r_{11} & =\lambda_{1} T(\sigma), \quad r_{12}=-\lambda_{12} T(\sigma), & r_{22} & =\lambda_{2} T(\sigma), \\
s_{11} & =f_{11} T(\sigma), & s_{12} & =f_{12} T(\sigma), & s_{13} & =f_{13} T(\sigma), \\
s_{22} & =f_{22} T(\sigma), & s_{23} & =-\frac{f_{32}+f_{23}}{2} T(\sigma)=-f_{23} T(\sigma), & s_{33} & =f_{33} T(\sigma),
\end{aligned}
$$

where $T(\sigma)$ was introduced in (1.3). When applying to a system near equilibrium, i.e. setting $T(\sigma)$ and $\mu(\sigma)$ to equilibrium values equations (2.31)-(2.33) reproduce precisely the standard fluctuation-dissipation relations, yet here they are derived for arbitrary $\tau\left(\sigma^{a}\right)$ and $\mu\left(\sigma^{a}\right)$ and thus are valid for far-from-equilibrium situations. ${ }^{8}$ As discussed below (2.19), the coefficients of (2.19) have to be such that right hand side of (2.19) is always non-negative. From relations (2.31)-(2.33), this translates into the statement that dissipative parameters such as the conductivity, shear and bulk viscosities are non-negative [1]. This reproduces the inequality-type constraints from the entropy current.

Note that to the orders of derivative expansion given in (2.9) and (2.19) there is no difference between quantum or classical regime. Neither are the results sensitive to $\theta_{0}$.

The local KMS prescription (1.14)-(1.15) works very well, it reproduces of all the known constraints on hydrodynamical equations, predicts new nonlinear Onsager relations as well as non-equilibrium fluctuation-dissipation relations. Yet there are still a number of deficiencies. Firstly there is an ambiguity in its formulation. A key element of (1.14) is to set the "background value" of $e^{\tau}$ to $\sqrt{-g_{r 00}}$ motivated from that in a curved spacetime with metric $g_{\mu \nu}$ the local proper temperature is proportional to $\frac{1}{\sqrt{-g_{00}}}$. The value $\sqrt{-g_{r 00}}$ was chosen for $e^{\tau}$ as it is symmetric in 1,2 and reduces to $\sqrt{-g_{00}}$ when $g_{1}=g_{2}=g$. The choice of is clearly not unique. For example, another possibility is ${ }^{9}$

$$
\tau=\frac{1}{4}\left(\log \left(-g_{100}\right)+\log \left(-g_{200}\right)\right) .
$$

Secondly, while there have been many supporting evidences that the prescription (1.14)-(1.15) indeed ensures the KMS condition (1.17), there has not been a general proof. Thirdly, the condition (1.17) should be physically equivalent regardless of choice of $\theta$ in (1.16). But in the the formulation (1.15) this is not clear. Finally, (1.15) is not formulated directly in the dynamical variables which makes its implementation and use

\footnotetext{
${ }^{8}$ Recall all the coefficients are functions of $\tau\left(\sigma^{a}\right)$ and $\mu\left(\sigma^{a}\right)$.

${ }^{9}$ Note that at the level of (2.9) and (2.19) using (2.34) does not make a difference.
} 
inconvenient. In particular it relies on the feature that in $I_{\text {hydro }}$ the dynamical variables and background fields always come together in the form of $h_{1,2}$ and $B_{1,2}$. This feature will likely not hold in the presence of anomalies.

In section 3 we discuss the classical limit of (1.4) and show that the first three issues mentioned in the previous paragraph are all addressed in that limit. In section 4 we introduce a dynamical formulation which acts directly on the dynamical variables.

\section{Classical limit}

The path integrals (1.4) describe macroscopic behavior of quantum systems with a nonzero $\hbar$. In this section we consider the classical limit $\hbar \rightarrow 0$. Note in the classical limit the path integrals (1.4) survive and describe classical statistical fluctuations.

\subsection{Small $\hbar$ expansion}

Following the discussion of [1], reinstating $\hbar$ we can write various background and dynamical fields as

$$
\begin{array}{rlrlrl}
g_{1 \mu \nu} & =g_{\mu \nu}+\frac{\hbar}{2} g_{a \mu \nu}, & g_{2 \mu \nu} & =g_{\mu \nu}-\frac{\hbar}{2} g_{a \mu \nu}, & A_{1 \mu} & =A_{\mu}+\frac{\hbar}{2} A_{a \mu}, \\
X_{1}^{\mu} & =X^{\mu}+\frac{\hbar}{2} X_{a}^{\mu}, & A_{2 \mu} & =A_{\mu}-\frac{\hbar}{2} A_{a \mu} \\
X_{2}^{\mu} & =X^{\mu}-\frac{\hbar}{2} X_{a}^{\mu}, & \varphi_{1} & =\varphi+\frac{\hbar}{2} \varphi_{a}, & \varphi_{2} & =\varphi-\frac{\hbar}{2} \varphi_{a}
\end{array}
$$

and $\theta, \beta_{0}$ in (1.16) become $\hbar \theta, \hbar \beta_{0}$. Furthermore, suppose $f^{(n)}$ is a coefficient in $n$-th order action $I^{(n)}$ in the $a$-field (noises) expansion (2.7), then $f^{(n)}$ can be expanded in $\hbar$ as

$$
f^{(n)}=\frac{1}{\hbar^{n-1}}\left(f_{\mathrm{cl}}^{(n)}+O(\hbar)+\cdots\right) .
$$

In (1.6)-(1.7), various transformation parameters can be written as

$$
f_{1}^{\mu}=f^{\mu}+\frac{1}{2} \hbar f_{a}^{\mu}, \quad f_{2}^{\mu}=f^{\mu}-\frac{1}{2} \hbar f_{a}^{\mu}, \quad \lambda_{1}=\lambda+\frac{1}{2} \hbar \lambda_{a}, \quad \lambda_{2}=\lambda-\frac{1}{2} \hbar \lambda_{a} .
$$

In the $\hbar \rightarrow 0$ limit, the two diffeomorphisms (1.6) then become: (i) physical space diffeomorphisms

$$
X^{\mu} \rightarrow X^{\prime \mu}(X)=f^{\mu}(X),
$$

under which $X_{a}^{\mu}$ transform as a vector, $g_{\mu \nu}, g_{a \mu \nu}$ as symmetric tensors, and $A_{\mu}, A_{a \mu}$ as one-forms, and (ii) noise diffeomorphisms under which various quantities transform as

$$
X_{a}^{\prime \mu}(\sigma)=X_{a}^{\mu}(\sigma)+f_{a}^{\mu}(X(\sigma)), \quad g_{a \mu \nu}^{\prime}=g_{a \mu \nu}-\mathcal{L}_{f_{a}} g_{\mu \nu}, \quad A_{a \mu}^{\prime}=A_{a \mu}-\mathcal{L}_{f_{a}} A_{\mu} .
$$

where $\mathcal{L}_{w}$ denotes Lie derivative along a vector $w^{\mu}$. We emphasize that (3.6) are finite transformations. The gauge transformations (1.7) become physical spacetime gauge transformation

$$
A_{\mu}^{\prime}=A_{\mu}-\partial_{\mu} \lambda(X), \quad A_{a \mu}^{\prime}(X)=A_{a \mu}(X)+\partial_{\mu}\left(\mathcal{L}_{X_{a}} \lambda\right), \quad \varphi^{\prime}(\sigma)=\varphi(\sigma)+\lambda(X(\sigma)),
$$


and noise gauge transformation

$$
A_{a \mu}^{\prime}(X)=A_{a \mu}(X)-\partial_{\mu} \lambda_{a}(X), \quad \varphi_{a}^{\prime}(\sigma)=\varphi_{a}(\sigma)+\lambda_{a}(X(\sigma)) .
$$

We then find that in this limit

$$
\begin{aligned}
h_{1 a b} & =\partial_{a} X_{1}^{\mu} \partial_{b} X_{1}^{\nu} g_{1 \mu \nu}\left(X_{1}\right)=h_{a b}(\sigma)+\frac{\hbar}{2} h_{a b}^{(a)}+O\left(\hbar^{2}\right), \\
B_{1 a} & =\partial_{a} X_{1}^{\mu} A_{1 \mu}\left(X_{1}\right)+\partial_{a} \varphi_{1}=B_{a}(\sigma)+\frac{\hbar}{2} B_{a}^{(a)}+O\left(\hbar^{2}\right),
\end{aligned}
$$

where

$$
\begin{aligned}
h_{a b}(\sigma) & \equiv \partial_{a} X^{\mu} \partial_{b} X^{\nu} g_{\mu \nu}(X), & h_{a b}^{(a)} & =\partial_{a} X^{\mu} \partial_{b} X^{\nu} G_{a \mu \nu}(X), \\
B_{a} & \equiv \partial_{a} X^{\mu} A_{\mu}(X)+\partial_{a} \varphi(\sigma), & B_{a}^{(a)} & =\partial_{a} X^{\mu} C_{a \mu}(X) \\
G_{a \mu \nu}(X) & \equiv g_{a \mu \nu}+\mathcal{L}_{X_{a}} g_{\mu \nu}, & C_{a \mu} & \equiv A_{a \mu}(X)+\partial_{\mu} \varphi_{a}(X)+\mathcal{L}_{X_{a}} A_{\mu}
\end{aligned}
$$

and $\varphi_{a}(X) \equiv \varphi_{a}(\sigma(X))$. It can be readily checked that $C_{a \mu}$ and $G_{a \mu \nu}$ are invariant under (3.6)-(3.8) and transform as a vector and tensor respectively under (3.5). The corresponding equations for $h_{2}, B_{2}$ are obtained from (3.9) by switching the signs before the $O(\hbar)$ terms. We now have

$$
\frac{1}{\hbar} I_{\text {hydro }}\left[h_{1}, B_{1} ; h_{2}, B_{2} ; \tau\right]=I_{\text {hydro }}\left[h_{a b}, B_{a} ; h_{a b}^{(a)}, B_{a}^{(a)} ; \tau\right]+O(\hbar) .
$$

As before decompose $h_{a b}$ and $B_{a}$ as

$$
\begin{aligned}
h_{a b} d \sigma^{a} d \sigma^{b} & =-b^{2}\left(d \sigma^{0}-v_{i} d \sigma^{i}\right)^{2}+a_{i j} d \sigma^{i} d \sigma^{j}, \\
B_{a} d \sigma^{a} & =\mu b\left(d \sigma^{0}-v_{i} d \sigma^{i}\right)+\mathfrak{b}_{i} d \sigma^{i} .
\end{aligned}
$$

and

$$
\frac{\partial X^{\mu}}{\partial \sigma^{0}} \equiv b u^{\mu}, \quad u^{\mu} u_{\mu}=-1, \quad u_{\mu}=g_{\mu \nu} u^{\nu}, \quad \frac{\partial X^{\mu}}{\partial \sigma^{i}} \equiv-v_{i} b u^{\mu}+\lambda_{i}{ }^{\mu}, \quad u_{\mu} \lambda_{i}{ }^{\mu}=0
$$

where $u^{\mu}$ is the local velocity field. We find various quantities in (2.3)-(2.5) become

$$
\begin{aligned}
& E_{r}=b, \quad a_{r i j}=a_{i j}, \quad V_{r i}=V_{i}=b v_{i} \quad \mathfrak{b}_{r i}=\mathfrak{b}_{i}, \quad \mu_{r}=\mu \\
& E_{a}=-\frac{1}{2} u^{\mu} u^{\nu} G_{a \mu \nu}(X), \quad V_{a i}=u^{\mu} \lambda_{i}^{\nu} G_{a \mu \nu}, \quad \Xi=\left(\lambda^{i \mu} \lambda_{j}^{\nu}-\frac{\Delta^{\mu \nu}}{d-1} \delta_{i}^{j}\right) G_{a \mu \nu}, \\
& \chi_{a}=\frac{1}{2} \Delta^{\mu \nu} G_{a \mu \nu} \quad \mu_{a}=u^{\mu} C_{a \mu}+\frac{1}{2} \mu u^{\mu} u^{\nu} G_{a \mu \nu}, \quad \mathfrak{b}_{a i}=\lambda_{i}^{\mu} C_{a \mu}+\mu u^{\mu} \lambda_{i}^{\nu} G_{a \mu \nu}
\end{aligned}
$$

and

$$
\nu_{a}=\mu_{a}+E_{a} \mu_{r}=u^{\mu} C_{a \mu}, \quad \mathfrak{c}_{a i}=\mathfrak{b}_{i}-\mu_{r} V_{a i}=\lambda_{i}^{\mu} C_{a \mu} .
$$

As anticipated from (3.10)-(3.12) all $a$-type fields can be obtained from $C_{a \mu}$ and $G_{a \mu \nu}$. 
Now let us consider the KMS condition (1.17)-(1.16) in the $\hbar \rightarrow 0$ limit. Equations (1.16) can be written as

$$
\tilde{\phi}(x)=\phi(-x), \quad \tilde{\phi}_{a}(x)=\phi_{a}(-x)+i \mathcal{L}_{\beta_{0}} \phi(-x), \quad \beta_{0}^{\mu}=\beta_{0}\left(\frac{\partial}{\partial x^{0}}\right)^{\mu}
$$

where $\phi=\left\{g_{\mu \nu}, A_{\mu}\right\}$ and $\phi_{a}=\left\{g_{a \mu \nu}, A_{a \mu}\right\}$. Note that parameter $\theta$ has dropped out. Equation (1.17) can be written as

$$
W\left[\phi(x), \phi_{a}(x)\right]=W\left[\phi(-x), \phi_{a}(-x)+i \mathcal{L}_{\beta_{0}} \phi(-x)\right] .
$$

We emphasize that in (3.22) the shift in $\phi_{a}$ is a finite transformation so one cannot expand the right hand side in $\mathcal{L}_{\beta_{0}} \phi(-x)$. Note that under (1.14) we have

$$
h_{a b}=g_{\mu \nu} \delta_{a}^{\mu} \delta_{b}^{\nu}, \quad h_{a b}^{(a)}=g_{a \mu \nu} \delta_{a}^{\mu} \delta_{b}^{\nu}, \quad B_{a}=A_{\mu} \delta_{\mu}^{a}, \quad B_{a}^{(a)}=A_{a \mu} \delta_{\mu}^{a}
$$

thus the local KMS prescription (1.15) implies that the action satisfies

$$
I_{\text {hydro }}\left[h_{a b}, B_{a} ; h_{a b}^{(a)}, B_{a}^{(a)} ; \tau\right]=I_{\text {hydro }}\left[\tilde{h}_{a b}, \tilde{B}_{a} ; \tilde{h}_{a b}^{(a)}, \tilde{B}_{a}^{(a)} ; \tilde{\tau}\right]
$$

where

$$
\begin{aligned}
& \tilde{h}_{a b}(\sigma)=h_{a b}(-\sigma), \quad \tilde{B}_{a}(\sigma)=B_{a}(-\sigma), \quad \tilde{\tau}(\sigma)=\tau(-\sigma) \\
& \tilde{h}_{a b}^{(a)}(\sigma)=h_{a b}^{(a)}(-\sigma)+i \beta_{0} \partial_{0} h_{a b}(-\sigma), \quad \tilde{B}_{a}^{(a)}(\sigma)=B_{a}^{(a)}(-\sigma)+i \beta_{0} \partial_{0} B_{a}(-\sigma) .
\end{aligned}
$$

From an argument given in appendix A we can then immediately conclude from (3.24) that the KMS condition (3.22) is satisfied at tree level.

\subsection{Physical space formulation}

In the $\hbar \rightarrow 0$ limit the physical spacetime formulation is much simplified. In fact the fluid and physical spacetime formulations become essentially the same. In this subsection, rather than starting from the fluid spacetime formulation we present an intrinsic formulation for the fluid action in the physical spacetime itself.

In the classical limit, in the physical spacetime the dynamical variables are $\sigma^{a}(x), \varphi(x)$, $\tau(x)$ and $X_{a}^{\mu}(x), \varphi_{a}(x)$. The background fields are $g_{\mu \nu}(x), A_{\mu}(x), g_{a \mu \nu}(x), A_{a \mu}(x)$ with $g_{\mu \nu}$ the physical spacetime metric. The action should be invariant under: (i) physical spacetime diffeomorphism (3.5); (ii) noise diffeomorphism (3.6); (iii) gauge transformation (3.7); (iv) noise gauge transformation (3.8); (v) time and spatial diffeomorphisms of $\sigma^{a}(1.8)-(1.9)$ which are now "global" symmetries; (vi) the diagonal shift (1.10) which is also now a global symmetry; (vii) equations (1.11) and (1.13) which are now imposed on physical spacetime action; (viii) the local KMS condition.

(ii)-(iv) imply that $a$-fields (including both background and dynamical variables) must appear in the combinations $G_{a \mu \nu}, C_{a \mu}$ introduced in (3.12), as these are the only combinations invariant under (3.6)-(3.8), while $A_{\mu}$ and $\varphi$ must appear through $B_{\mu}=A_{\mu}+\partial_{\mu} \varphi(x)$. By using the time diffeomorphism (1.9) we can set $\sigma^{0}=x^{0}$. In the absence of parity or 
time reversal breaking, invariance under (1.8) implies that the only invariant which can be constructed is the velocity field $u^{\mu}$ defined by

$$
u^{\mu}=\frac{1}{\sqrt{-j^{2}}} j^{\mu}, \quad j^{2} \equiv j^{\mu} j_{\mu}, \quad j^{\mu}=\epsilon^{\mu \mu_{1} \cdots \mu_{d-1}} \frac{\partial \sigma^{1}}{\partial x^{\mu_{1}}} \cdots \frac{\partial \sigma^{d-1}}{\partial x^{\mu_{d-1}}}
$$

where $\epsilon$ is the antisymmetric tensor and indices are raised and lowered by $g_{\mu \nu}$ and its inverse. Note that $j^{2}$ is not invariant under (1.8) and by definition

$$
u^{\mu} u_{\mu}=-1
$$

It can be readily checked the definition (3.27) coincides with that in (3.16). $B_{\mu}$ is not invariant under shift (1.10) of $\varphi$, but

$$
\mu \equiv u^{\mu} B_{\mu}, \quad F_{\mu \nu}=\partial_{\mu} B_{\nu}-\partial_{\nu} B_{\mu}
$$

are invariant. Note that $F_{\mu \nu}$ does not depend on the dynamical variables.

To summarize, the only combinations of $r$-variables which can appear are

$$
\beta(x)=\beta_{0} e^{\tau(x)}, \quad u^{\mu}, \quad \mu, \quad F_{\mu \nu}, \quad g_{\mu \nu} .
$$

Sometimes it is convenient to combine the first three variables further into

$$
\beta^{\mu}=\beta(x) u^{\mu}(x), \quad \hat{\mu}(x)=\beta(x) \mu=\beta^{\mu}(x) B_{\mu}
$$

where $\beta^{\mu}$ is now unconstrained. Any scalar functions in the action must only depend on $\mu$ and $\beta(x)$.

Now introducing notation

$$
G_{a \mu M}=\left(G_{a \mu \nu}, 2 C_{a \mu}\right), \quad M=(\mu, d), \quad G_{a \mu d}=2 C_{a \mu}
$$

we can write the full action as

$$
I_{\text {hydro }}=\int d^{d} x \sqrt{-g} \mathcal{L}
$$

with

$$
\mathcal{L}=\sum_{n=1}^{\infty} \mathcal{L}^{(n)}=\sum_{n=1}^{\infty} i^{\eta_{n}} f^{(n)}\left[\Lambda_{r}\right] G_{a}^{n}, \quad \eta_{n}= \begin{cases}1 & n \text { even } \\ 0 & n \text { odd }\end{cases}
$$

where $\Lambda_{r}$ denotes the collection $\Lambda_{r}=\left\{\beta^{\mu}, \hat{\mu}, F_{\mu \nu}, g_{\mu \nu}\right\}$ and we have suppressed all spacetime indices. The $n$-th term in (3.34) should be understood as

$$
f^{(n)}\left[\Lambda_{r}\right] G_{a}^{n}=f_{\mu_{1} \cdots \mu_{n}, M_{1}, \cdots M_{N}}^{(n)}\left(\Lambda_{r} ; \partial_{\mu}\right) G_{a \mu_{1} M_{1}}(x) \cdots G_{a \mu_{n} M_{n}}(x)
$$

where the notation $f_{\mu_{1} \cdots \mu_{n}, M_{1}, \cdots M_{N}}^{(n)}\left(\Lambda_{r} ; \partial_{\mu}\right)$ indicate it is a function of $\Lambda_{r}$, their derivatives, as well as derivative operators acting on $G_{a \mu M}$. The whole action should be diffeomorphism invariant. The first few terms can be written explicitly as

$$
\mathcal{L}=\frac{1}{2} T^{\mu M} G_{a \mu M}+\frac{i}{4} W^{\mu \nu, M N} G_{a \mu M} G_{a \nu N}+\frac{1}{8} Y^{\mu \nu \rho, M N P} G_{a \mu M} G_{a \nu N} G_{a \rho P}+\cdots,
$$


with

$$
\frac{1}{2} T^{\mu M} G_{a \mu M}=T^{\mu \nu}\left(\frac{1}{2} g_{a \mu \nu}+\nabla_{\mu} X_{a \nu}\right)+J^{\mu}\left(A_{\mu a}+\partial_{\mu} \varphi_{a}+X_{a}^{\nu} \nabla_{\nu} A_{\mu}+A_{\nu} \nabla_{\mu} X_{a}^{\nu}\right) .
$$

In the first term of (3.36) by integrating by part we can move all the derivatives on $G_{a \mu M}$ into $T^{\mu M}=\left(T^{\mu \nu}, J^{\mu}\right)$. One should keep in mind that $W, Y$ still contain derivatives on $G$ 's. From coupling to $g_{a \mu \nu}$ and $A_{a \mu}$ we can thus identify $T^{\mu \nu}$ and $J^{\mu}$ as the hydrodynamic stress tensor and $\mathrm{U}(1)$ current.

Note that in derivative counting, $u^{\mu}, \mu, \beta, G_{a \mu M}$ should all be counted as zeroth order.

In the absence of $g_{a \mu \nu}$ and $A_{a \mu}$, the equations of motion of (3.34) by varying with respect to $r$-variables can be consistently solved by setting $X_{a}^{\mu}=\varphi_{a}=0$. The nontrivial equations of motion arise from varying with respect to $X_{a}^{\mu}$ and $\varphi_{a}$, and only the first term in (3.36) is relevant leading to

$$
\nabla_{\nu} T^{\mu \nu}-F^{\mu \nu} J_{\nu}=0, \quad \nabla_{\mu} J^{\mu}=0 .
$$

The action (3.36) can also be reached by starting from the fluid spacetime action and inverting $X^{\mu}(\sigma)$. In particular, applying discussion parallel to that of appendix $\mathrm{F}$ of [1] one can prove that all coefficients in (3.36) can indeed be expressed only in terms of $\beta^{\mu}, \hat{\mu}, F_{\mu \nu}, g_{\mu \nu}$. The implications from the local KMS condition discussed earlier in section 2.3 for the fluid spacetime action can also be translated to (3.36). In section 5 we will present an alternative way to work out those constraints using the new dynamical formulation introduced in next section.

Now let us comment on the relation with previous literature. $G_{a \mu \nu}$ and $C_{a \mu}$ already appeared in [12] $]^{10}$ as well as the $O(a)$ part of the action (3.36). But in [12] it was not clear how to extend the action beyond $O(a)$ at nonlinear level. Here we show that these quantities are in fact exact. Also local KMS condition was not discussed there.

The physical spacetime formulation (in the gauge $\sigma^{0}=x^{0}$ ) shares some common elements with the formulation of [2-11], in addition to having noise fields $X_{a}^{\mu}, \varphi_{a}$, the key differences are that: (i) in (1.8) we require general spatial diffeomorphisms while in [2-11] only volume-preserving diffeomoprhisms are allowed; (ii) we have an additional scalar field $\tau$ which serves as local temperature. Suppose we only require volume-preserving diffeomoprhisms in (1.8), then $\sqrt{-j^{2}}$ in (3.27) is invariant and becomes a dynamical variable which naively may be used to replace $\tau$. However, by definition $j^{\mu}$ is exactly conserved regardless of the presence of dissipation. Such a conserved quantity appears to have no place in dissipative hydrodynamics. In our construction the full spatial diffeomorphisms get rid of $j^{2}$, and we supplement that by introducing $\tau$. The resulting $\beta^{\mu}$ is then unconstrained.

\section{Dynamical KMS symmetry}

In this section we introduce an alternative to the local KMS condition. The new formulation, to which we refer as dynamical KMS condition (or symmetry), directly acts on dynamical variables. We first discuss the proposal at finite $\hbar$ in the fluid spacetime and then discuss the classical limit. At the end we discuss some open issues at quantum level.

\footnotetext{
${ }^{10} G_{a \mu \nu}$ is equal to $\xi_{\mu \nu}^{a}$, which is defined at the beginning of section 4.2 , and $C_{a \mu}$ is $\chi_{\mu}^{a}$, which is defined below eq. (4.16) there.
} 


\subsection{Proposal}

We propose that in the absence of background fields, the action $I_{\text {hydro }}$ is invariant under the following transformations on the dynamical variables $(s=1,2)$

$$
\tilde{X}_{s}^{\mu}(-\sigma)=-\Phi_{i \eta_{s}}^{*} X_{s}^{\mu}(\sigma)-i \eta_{s} \delta_{0}^{\mu}, \quad \tilde{\varphi}_{s}(-\sigma)=-\Phi_{i \eta_{s}}^{*} \varphi_{s}(\sigma), \quad \tilde{\tau}(\sigma)=\tau(-\sigma)
$$

with $\eta_{1}=-\theta, \eta_{2}=\beta_{0}-\theta$. In (4.1), $\Phi_{\lambda}$ is a one-parameter $(\lambda)$ diffeomorphism generated by vector field

$$
w^{a}=\frac{e^{\tau}}{b_{r}}\left(\frac{\partial}{\partial \sigma^{0}}\right)^{a}, \quad b_{r}=\sqrt{-h_{00}}
$$

and $\Phi_{\lambda}^{*}$ denotes its push-forward map. To implement (4.1) one needs to analytically continue $\Phi_{\lambda}^{*}$ to complex values of $\lambda$. In (4.1) the constant shifts in $X_{s}^{\mu}$ are chosen so that in the presence of background fields, if we transform the background fields as (1.16), $h_{\text {sab }}$ and $B_{s a}$ transform as

$$
\tilde{h}_{s a b}(\sigma)=\Phi_{i \eta_{s}}^{*} h_{s a b}(-\sigma), \quad \tilde{B}_{s a}(\sigma)=\Phi_{i \eta_{s}}^{*} B_{s a}(-\sigma),
$$

and the fluid spacetime action is invariant

$$
I_{\text {hydro }}\left[h_{1}, B_{1} ; h_{2}, B_{2}, \tau\right]=I_{\text {hydro }}\left[\tilde{h}_{1}, \tilde{B}_{1} ; \tilde{h}_{2}, \tilde{B}_{2} ; \tilde{\tau}\right] .
$$

Now using a general result in appendix A we immediately conclude that (4.1) ensures (1.17) at tree-level of the path integral (1.4). To ensure (1.17) at the level of the full path integral (i.e. including all loops), one needs to extend transformations (4.1) to fermionic fields, which will be discussed elsewhere.

In (4.2) the factor $1 / b_{r}$ is inserted so that $w^{a}$ is independent of choice of $\sigma^{0} . \Phi_{\lambda}$ is a time diffeomorphism which can be written as

$$
u_{\lambda}^{0} \equiv u^{0}\left(\sigma^{a} ; \lambda\right), \quad u^{i}=\sigma^{i}
$$

where $u^{0}\left(\sigma^{a} ; \lambda\right)$ is obtained by solving the differential equation

$$
\frac{d u^{0}}{d \lambda}=\frac{e^{\tau}}{b_{r}}\left(u^{a}\right), \quad u^{0}(\lambda=0)=\sigma^{0} .
$$

We then have

$$
\Phi_{\lambda}^{*} X_{s}^{\mu}(\sigma)=X_{s}^{\mu}\left(u_{-\lambda}^{a}(\sigma)\right), \quad \Phi_{\lambda}^{*} \varphi_{s}(\sigma)=\varphi_{s}\left(u_{-\lambda}^{a}(\sigma)\right) .
$$

At a heuristic level, one may interpret the action of $\Phi_{i \eta_{s}}^{*}$ as shifting $\hat{\sigma}^{0}$ by $i \eta_{s} e^{\tau}$ where $\hat{\sigma}^{0}$ is a proper time defined by $d \hat{\sigma}^{0}=b_{r} d \sigma^{0}$. We stress that, as in (1.14), it is the appearance of $e^{\tau}$ in (4.2) that "defines" it as the local inverse temperature. In other words, if we had used some other function of $\tau$ in (4.2) then it would be that function which should be identified as the local temperature.

We should mention that if we replace $\Phi_{i \eta_{s}}^{*}$ in (4.1) by $\Phi_{\lambda_{s}}^{*}$ with some arbitrary parameter $\lambda_{s}$, then one will still get (4.3) with again $i \eta_{s}$ replaced by $\lambda_{s}$. Furthermore one can still use the argument of appendix A to conclude that (4.1) ensures (1.17) at tree-level of 
the path integral (1.4). We now show that $\lambda_{s}$ are in fact required to be $i \eta_{s}$ for $X_{s}^{\mu}$ to have the right boundary conditions. We require at spacetime infinities both the background and dynamical fields go to zero, i.e. at spacetime infinities, the physical and fluid spacetime coincide and the system is in thermal equilibrium. Write

$$
X^{\mu}(\sigma)=\sigma^{a} \delta_{a}^{\mu}+\pi^{\mu}(\sigma)
$$

then $\pi^{\mu}$ (which does not have to be small) should go to zero at the spacetime infinities of the fluid spacetime. In (4.6) as $u^{a} \rightarrow \infty$, we should have $w^{0} \rightarrow 1$ and thus

$$
u_{\lambda}^{0}(\sigma \rightarrow \infty)=\sigma^{0}+\lambda, \quad u^{i}=\sigma^{i} .
$$

Now using (4.7) and (4.9) we thus find that if we use $\Phi_{\lambda_{s}}$ in (4.1)

$$
\tilde{X}_{s}^{\mu}(\sigma \rightarrow \infty)=-u_{-\lambda_{s}}^{a}(-\sigma) \delta_{a}^{\mu}-i \eta_{s} \delta_{0}^{\mu}=\sigma^{a} \delta_{a}^{\mu}+\left(\lambda_{s}-i \eta_{s}\right) \delta_{0}^{\mu}, \quad \sigma \rightarrow \infty .
$$

We thus conclude

$$
\lambda_{s}=i \eta_{s}
$$

\subsection{The classical limit}

Let us look at transformations (4.1) in the classical limit $\hbar \rightarrow 0$. With the notations introduced in section 3 we find ${ }^{11}$

$$
\begin{aligned}
\tilde{X}^{\mu}(\sigma) & =-X^{\mu}(-\sigma), & \tilde{X}_{a}^{\mu}(\sigma) & =-X_{a}^{0}(-\sigma)-i \beta^{\mu}(-\sigma)+i \beta_{0}^{\mu} \\
\tilde{\varphi}(\sigma) & =-\varphi(-\sigma), & \tilde{\varphi}_{a}(\sigma) & =-\varphi_{a}(-\sigma)-i \beta^{a} \partial_{a} \varphi(-\sigma)
\end{aligned}
$$

where again $\theta$ has dropped out and

$$
\beta(\sigma)=\beta_{0} e^{\tau(\sigma)}, \quad \beta^{a} \equiv \beta_{0} w^{a}, \quad \beta^{\mu}=\beta(\sigma) u^{\mu}(\sigma)=\partial_{a} X^{\mu} \beta^{a} .
$$

We then find that

$$
\begin{aligned}
& \tilde{h}_{a b}(\sigma)=h_{a b}(-\sigma), \quad \tilde{h}_{a b}^{(a)}(\sigma)=h_{a b}^{(a)}(-\sigma)+i \mathcal{L}_{\beta} h_{a b}(-\sigma) \\
& \tilde{B}_{a}(\sigma)=B_{a}(-\sigma), \quad \tilde{B}_{a}^{(a)}(\sigma)=B_{a}^{(a)}(-\sigma)+i \mathcal{L}_{\beta} B_{a}(-\sigma)
\end{aligned}
$$

where $\mathcal{L}_{\beta}$ is the Lie derivative along vector $\beta^{a}$.

Note that one can use the time diffeomorphism (1.9) to set

$$
\sqrt{-h_{00}}=e^{\tau}
$$

in which case

$$
\beta^{a}=\beta_{0}\left(\frac{\partial}{\partial \sigma^{0}}\right)^{a}
$$

\footnotetext{
${ }^{11}$ We note that despite some resemblance of eqs. (4.12) and (4.15) with the $\mathrm{U}(1)_{T}$ transformation of [17] (see e.g. eq. (5.2) there), they are fundamentally different. Here due to an additional spacetime reflection, the transformation is a discrete $Z_{2}$ transformation. Many consequences of the theory depend crucially on this feature. In contrast a U(1) transformation will lead to completely different (physically inconsistent) results.
} 
and then the Lie derivatives in (4.15)-(4.16) become ordinary derivatives along the time direction in the fluid spacetime. Equation (4.17) is a constraint between local temperature and $X^{\mu}(\sigma)$ which makes them no longer independent.

One can readily check that the above transformation is $Z_{2}$. The above equations can be written more explicitly in terms of various components as

$$
\tilde{\Phi}(\sigma)=\Phi(-\sigma), \quad \text { for } \quad \Phi=\left\{b, v_{i}, a_{i j}, \mu, \mathfrak{b}_{i}\right\}
$$

and

$$
\begin{array}{rlrl}
\tilde{E}_{a}(-\sigma) & =E_{a}(\sigma)+i \beta(\sigma) D_{0} \tau(\sigma), & \tilde{\chi}_{a}(-\sigma) & =\chi_{a}(\sigma)+i \beta(\sigma) D_{0} \log \sqrt{a}(\sigma) \\
\tilde{\Xi}(-\sigma) & =\tilde{\Xi}(\sigma)+i \beta(\sigma)\left(a^{-1} D_{0} a\right)_{\text {traceless }}(\sigma), & \tilde{\mathfrak{b}}_{a i}(-\sigma)=\mathfrak{b}_{a i}(\sigma)+i \beta(\sigma) D_{0} \mathfrak{b}_{i}(\sigma),(4.21) \\
\tilde{\mu}_{a}(-\sigma) & =\mu_{a}(\sigma)+i \beta(\sigma) D_{0} \mu(\sigma), & \tilde{V}_{a i}(-\sigma) & =V_{a i}(\sigma)+i \beta(\sigma)\left(D_{i} E(\sigma)-D_{i} \tau(\sigma)\right) \\
\tilde{\nu}_{a}(-\sigma) & =\nu_{a}(\sigma)+i D_{0} \hat{\mu}(\sigma), & \mathfrak{c}_{a i}(-\sigma) & =\mathfrak{c}_{a i}(\sigma)+i\left(\beta \hat{D}_{0} \mathfrak{b}_{i}+\hat{\mu} D_{i} \tau\right) .
\end{array}
$$

Note that with $e^{\tau}=\sqrt{-g_{r 00}}$, the vector field (4.2) is given by

$$
w^{0}=1+O(\hbar)
$$

and equations (4.15)-(4.16) become (3.25)-(3.26). We thus conclude that in the classical limit, the new prescription is precisely equivalent to (1.14)-(1.15). As a cross check, applying (4.4) with (4.15)-(4.16) to (2.9) and (2.19) we find indeed identical results to $(2.25)-(2.33)$.

Finally let us write down the dynamical KMS transformation for fields in physical spacetime. From (4.12)-(4.13) we immediately have

$$
\begin{aligned}
\tilde{\sigma}^{i}(x) & =-\sigma^{i}(-x), & \tilde{\tau}(x) & =\tau(-x), \quad \tilde{\varphi}(x)=-\varphi(-x), \\
\tilde{X}_{a}^{\mu}(-x) & =-X_{a}^{\mu}(x)-i \beta^{\mu}(x)+i \beta_{0}^{\mu}, & \tilde{\varphi}_{a}(-x) & =-\varphi_{a}(x)-i \beta^{\mu} \partial_{\mu} \varphi(x)
\end{aligned}
$$

and thus

$$
\begin{aligned}
\tilde{u}^{\mu}(x) & =u^{\mu}(-x), \quad \tilde{\hat{\mu}}(x)=\hat{\mu}(-x), & \tilde{\beta}^{\mu}(x) & =\beta^{\mu}(-x), \\
\tilde{G}_{a \mu \nu}(-x) & =G_{a \mu \nu}(x)+\mathcal{L}_{i \beta^{\mu}} g_{\mu \nu}(x), & \tilde{C}_{a \mu}(-x) & =C_{a \mu}(x)+\mathcal{L}_{i \beta^{\mu}} B_{\mu}(x) .
\end{aligned}
$$

The above transformations are again $Z_{2}$. Using the unified notations of (3.32) we can also write (4.28) as

$$
\tilde{G}_{a \mu M}(-x)=G_{a \mu M}(x)+\mathcal{L}_{i \beta^{\mu}} g_{\mu M}(x)=G_{a \mu M}(x)+i \Phi_{r \mu M}
$$

where $g_{\mu M}=\left(g_{\mu \nu}, 2 B_{\mu}\right)$ and

$$
\Phi_{r \mu \nu}=\nabla_{\mu} \beta_{\nu}+\nabla_{\nu} \beta_{\mu}, \quad \Phi_{r \mu d}=2 \mathcal{L}_{\beta^{\mu}} B_{\mu}(x)=2\left(\nabla_{\mu} \hat{\mu}-\beta^{\nu} F_{\mu \nu}\right)
$$




\subsection{Open issues at finite $\hbar$}

As in the prescription for $e^{\tau}$ in (1.14), here the choice of $1 / b_{r}$ factor in (4.2) is not unique. Other than that we need something to make $w^{a}$ independent of choice of $\sigma^{0}$, we do not have other criterion to fix it further at the moment. For example, instead of using $b_{r}$ we could have used $\sqrt{b_{1} b_{2}}$ or even use $b_{1}$ for $X_{1}$ and $b_{2}$ for $X_{2}$. In the classical limit all these choices become the same and there is a unique $b$. In the classical limit $\theta$ drops out and the dynamical KMS transformation is a $Z_{2}$ symmetry of the action. At finite $\hbar$, under (4.2) it does not appear that (4.1) is a $Z_{2}$ transformation, and it is also not clear whether different $\theta$ yields the same physics.

\section{Dynamical KMS invariance and entropy current}

To elucidate further the structure of the action for fluctuating hydrodynamics at classical level, in this section we work out the implications of invariance of (3.34) under dynamical KMS transformations (4.27)-(4.28). We will also explicitly construct the entropy current to first derivative order using the procedure of [28].

\subsection{Dynamical KMS invariance}

Under a dynamical KMS transformation (4.27)-(4.29) we find (3.34) becomes

$$
\tilde{\mathcal{L}}=\sum_{n=1}^{\infty} \widetilde{\mathcal{L}^{(n)}}=\sum_{n=1}^{\infty} i^{\eta_{n}} f^{(n) *}\left[\Lambda_{r}\right]\left(G_{a}+i \Phi_{r}\right)^{n}
$$

where $f^{(n) *}$ is obtained from $f^{(n)}$ with a sign flip on derivatives

$$
f_{\alpha_{1} \cdots \alpha_{n}}^{(n) *}\left(\Lambda_{r}(x) ; \partial_{\mu}\right)=f_{\alpha_{1} \cdots \alpha_{n}}^{(n)}\left(\Lambda_{r}(x) ;-\partial_{\mu}\right) .
$$

The dynamical KMS condition can then be written as

$$
\mathcal{L}=\tilde{\mathcal{L}}-\nabla_{\mu} V^{\mu}
$$

Taking another tilde operation on the above equation and from its $Z_{2}$ nature we find that

$$
\tilde{V}^{\mu}=V^{\mu}
$$

where the tilde operation on $V^{\mu}$ should understood as in (5.1), i.e. one replaces $G_{a}$ by $G_{a}+i \Phi_{r}$ and then flips the sign of all the derivatives. Note that this is slightly different from the tilde operation (4.25)-(4.29) defined for individual fields. Below throughout the paper tilde operations on a quantity which is part of a Lagrangian should always be understood this way.

We can expand $V^{\mu}$ in terms of the number of $a$-fields and derivatives

$$
V^{\mu}=\sum_{n=0}^{\infty} V_{n}^{\mu}=\sum_{n, m=0}^{\infty} i^{\eta_{n}} V_{(n, m)}^{\mu}
$$


where $V_{n}^{\mu}$ contains $n$ factors of $G_{a}$, and $V_{(n, m)}^{\mu}$ contains $n$ factors of $G_{a}$ and $m$ derivatives. Equation (5.3) can then be written order by order in $a$-expansion as

$$
\mathcal{L}^{(n)}=(\tilde{\mathcal{L}})_{n}-\partial_{\mu} V_{n}^{\mu}
$$

where $(\tilde{\mathcal{L}})_{n}$ denotes $O\left(a^{n}\right)$ terms in $\tilde{\mathcal{L}}$. In particular, for $n=0$ we have

$$
(\tilde{\mathcal{L}})_{0}=\partial_{\mu} V_{0}^{\mu}
$$

Since $\Phi_{r}$ contains one derivative, the dynamical KMS condition (5.3) couple $n$-th derivative terms in $f^{(1)}$ with $(n-1)$-th derivative terms in $f^{(2)},(n-2)$-th derivative terms in $f^{(3)}$, etc. all the way to zeroth derivative terms in $f^{(n)}$. More explicitly, using the notation of $(2.8), \mathcal{L}^{(n, m)}$ with a fixed $l=n+m$ couple to one another. Introducing

$$
\mathcal{L}=\sum_{n=1}^{\infty} \sum_{m=0}^{\infty} \mathcal{L}^{(n, m)}=\sum_{l=1}^{\infty} \mathcal{L}_{l}, \quad \mathcal{L}_{l}=\sum_{n+m=l} \mathcal{L}^{(n, m)}, \quad V^{\mu}=\sum_{l=0}^{\infty} v_{l}^{\mu}, \quad v_{l}^{\mu}=\sum_{n+m=l} i^{\eta_{n}} V_{(n, m)}^{\mu}
$$

we then find that $(5.3)$ reduces to $\mathcal{L}_{l}$ being separately invariant

$$
\tilde{\mathcal{L}}_{l}-\mathcal{L}_{l}=\partial_{\mu} v_{l-1}^{\mu}, \quad l=1,2, \cdots
$$

When one considers a truncation of $\mathcal{L}$ in derivative and $a$-expansion one should do it in terms of $\mathcal{L}_{l}$ to be compatible with the dynamical KMS symmetry.

There is a simple way to impose dynamical KMS invariance (5.6) at order $O\left(a^{n}\right)$ with $n \geq 1$, which also shows that one can set $V_{n}^{\mu}$ with $n \geq 1$ to zero by absorbing such total derivatives into the definition of the Lagrangian. ${ }^{12}$ For this purpose let us consider a Lagrangian density $\mathcal{L}_{c}$ of the form (3.34), then due to $Z_{2}$ nature of the dynamical KMS transformations,

$$
\mathcal{L}=\frac{1}{2}\left(\mathcal{L}_{c}+\tilde{\mathcal{L}}_{c}\right)
$$

where $\tilde{\mathcal{L}}_{c}$ is defined as in (5.1), automatically satisfies dynamical KMS invariance. Note, however, that $\tilde{\mathcal{L}}_{c}$ contains terms with $r$-fields only, and the resulting $\mathcal{L}$ violates the condition (1.13). We must then further require that $O\left(a^{0}\right)$ terms in $\tilde{\mathcal{L}}_{c}$ be equal to zero, which is simply (5.7). Thus the combination of (5.10) and (5.7) is enough to ensure (5.3).

With a $\mathcal{L}$ built from (5.10), only $V_{0}^{\mu}$ is nonzero. In particular, from (5.4) $V_{0}^{\mu}$ should contain only even derivative terms as odd derivative terms change sign under tilde operation at $O\left(a^{0}\right)$. Furthermore, one can show that the even derivative part of (5.7) is automatically implied by (5.10) and thus one needs to consider only the odd derivative part of (5.7). Now using (5.9) we can write (5.7) more explicitly as

$$
\left(\tilde{\mathcal{L}}_{2 n+1}\right)_{0}=\left(\widetilde{\mathcal{L}^{(1,2 n)}}+\widetilde{\mathcal{L}^{(2,2 n-1)}}+\cdots+\widetilde{\mathcal{L}^{(2 n+1,0)}}\right)_{0}=i \nabla_{\mu} V_{(0,2 n)}^{\mu}, \quad n=1,2, \cdots
$$

\footnotetext{
${ }^{12}$ See appendix B for an alternative argument.
} 
or in terms of notations of (3.34)

$$
\sum_{k=1}^{2 n+1} \epsilon_{k} f^{(k, 2 n+1-k)}\left[\Lambda_{r}\right] \Phi_{r}^{k}=i \nabla_{\mu} V_{(0,2 n)}^{\mu}
$$

where $\epsilon_{k}=(-1)^{m}$ for $k=2 m+1,2 m+2$ and $f^{(k, l)}$ denotes terms with $l$ derivatives.

After imposing (5.10) and (5.7) we will need to perform a further integration by parts for $f^{(1)}$ terms in the Lagrangian (3.34) so that in the first term of (3.36) there is no derivative acting on $G$. This can generate a nonzero $V_{1}^{\mu}$.

As an illustration of the procedures outlined above, let us consider (3.36) up to

$$
\mathcal{L}=\mathcal{L}_{1}+\mathcal{L}_{2}+\mathcal{L}_{3}+\cdots
$$

i.e. to two derivatives in $T^{\mu M}$, one derivative in $W^{\mu \nu, M N}$, and zero derivative in $Y^{\mu \nu \rho, M N P}$. We will use the notation $T_{n}^{\mu M}$ to denote terms in $T^{\mu M}$ which contain $n$ derivatives, and similarly for others. We thus have

$$
\begin{aligned}
\mathcal{L}_{1} & =\frac{1}{2} T_{0}^{\mu M} G_{a \mu M}, \quad \mathcal{L}_{2}=\frac{1}{2} T_{1}^{\mu M} G_{a \mu M}+\frac{i}{4} W_{0}^{\mu \nu, M N} G_{a \mu M} G_{a \nu N}, \\
\mathcal{L}_{3} & =\frac{1}{2} T_{2}^{\mu M} G_{a \mu M}+\frac{i}{4} W_{1}^{\mu \nu, M N} G_{a \mu M} G_{a \nu N}+\frac{1}{8} Y_{0}^{\mu \nu \rho, M N P} G_{a \mu M} G_{a \nu N} G_{a \rho P} .
\end{aligned}
$$

Applying (5.10) we find

$$
T_{1}^{\mu M}=-\frac{1}{2} W_{0}^{\mu \nu, M N} \Phi_{r \mu N}, \quad W_{1}^{\mu \nu, M N}=\frac{3}{4} Y_{0}^{\mu \nu \rho, M N P} \Phi_{r \rho P} .
$$

We next turn to $O\left(a^{0}\right)$ dynamical KMS condition (5.12) which can be written explicitly as

$$
\frac{1}{2} T^{\mu M *} \Phi_{r \mu M}-\frac{1}{4} W^{\mu \nu, M N *} \Phi_{r \mu M} \Phi_{r \nu N}-\frac{1}{8} Y^{\mu \nu \rho, M N P *} \Phi_{r \mu M} \Phi_{r \nu N} \Phi_{r \rho P}+\cdots=\nabla_{\mu} V_{0}^{\mu} .
$$

At first order derivative order (5.17) gives

$$
\frac{1}{2} T_{0}^{\mu M} \Phi_{r \mu M}=\nabla_{\mu} V_{(0,0)}^{\mu}
$$

where the second subscript of $V$ denotes the number of derivatives. At second derivative order (5.17) is automatically satisfied from (5.16) with $V_{(0,1)}^{\mu}=0$, and at third derivative order using (5.16) we have

$$
\frac{1}{2} T_{2}^{\mu M} \Phi_{r \mu M}+\frac{1}{16} Y_{0}^{\mu \nu \rho, M N P} \Phi_{r \mu M} \Phi_{r \nu N} \Phi_{r \rho P}=\nabla_{\mu} V_{(0,2)}^{\mu} .
$$

Note that the above expression also indicates that to second order in $T^{\mu M}$ there is no derivative acting on $G_{a \mu M}$ in (3.36). So there is no need to do further integration-by-parts and to the current order $V_{1}^{\mu}=0$.

In [28] we showed for any theory of the form (3.34) (in the absence of $a$-type sources $g_{a \mu \nu}$ and $\left.A_{a \mu}\right)$ which satisfies (1.12) and is invariant under dynamical KMS transformations (4.27)-(4.28), there exists a current $S^{\mu}$ whose divergence is non-negative. From [28], to second order in derivative expansion $S^{\mu}$ can be written as

$$
S^{\mu}=V_{(0,0)}^{\mu}+V_{(0,2)}^{\mu}-T^{\mu \nu} \beta_{\nu}-J^{\mu} \hat{\mu} .
$$


Below we will work out its explicit form to first derivative order for a general charged fluid and show it indeed reproduces the standard entropy current. In next section we will work out its explicit expression at second derivative order for a conformal neutral fluid.

\subsection{Explicit tensor analysis to first order in derivative expansion}

We now expand various tensors above explicitly in terms of $\tau(x), u^{\mu}, \hat{\mu}, g_{\mu \nu}, F_{\mu \nu}$. The analysis becomes rather tedious at second order in derivatives for $T^{\mu M}$. So we will only write down the explicit expressions for $T^{\mu M}$ to first order in derivatives. In section 7 we give the explicit expression at second derivative orders for a conformal neutral fluid.

To first derivative order the most general $T^{\mu M}=\left(T^{\mu \nu}, J^{\mu}\right)$ can be written as

$$
T^{\mu \nu}=\epsilon u^{\mu} u^{\nu}+p \Delta^{\mu \nu}+2 u^{(\mu} q^{\nu)}+\Sigma^{\mu \nu}, \quad J^{\mu}=n u^{\mu}+j^{\mu},
$$

with

$$
\begin{aligned}
\epsilon & =\epsilon_{0}+h_{\epsilon}, \quad p=p_{0}+h_{p}, \quad \Sigma^{\mu \nu}=-\eta \sigma^{\mu \nu}, \quad n=n_{0}+h_{n}, \quad \Delta^{\mu \nu}=\eta^{\mu \nu}+u^{\mu} u^{\nu} \\
h_{\epsilon} & =f_{11} \partial \tau+f_{12} \theta+f_{13} \beta^{-1}(x) \partial \hat{\mu} \\
h_{p} & =f_{21} \partial \tau-f_{22} \theta+f_{23} \beta^{-1}(x) \partial \hat{\mu} \\
h_{n} & =f_{31} \partial \tau+f_{32} \theta-f_{33} \beta^{-1}(x) \partial \hat{\mu} \\
j^{\mu} & =\lambda_{21} \partial u^{\mu}-\lambda_{2}\left(\Delta^{\mu \nu} \partial_{\nu} \mu+u_{\lambda} F^{\lambda \mu}\right)+\lambda_{7} \Delta^{\mu \nu} \partial_{\nu} \tau+\lambda_{8} \Delta^{\mu \nu} \partial_{\nu} \mu \\
q^{\mu} & =-\lambda_{1} \partial u^{\mu}+\lambda_{12}\left(\Delta^{\mu \nu} \partial_{\nu} \mu+u_{\lambda} F^{\lambda \mu}\right)+\lambda_{5} \Delta^{\mu \nu} \partial_{\nu} \tau+\lambda_{6} \Delta^{\mu \nu} \partial_{\nu} \mu, \\
\partial & \equiv u^{\mu} \nabla_{\mu}, \quad \theta \equiv \nabla_{\mu} u^{\mu}, \quad \sigma^{\mu \nu} \equiv \Delta^{\mu \lambda} \Delta^{\nu \rho}\left(\nabla_{\lambda} u_{\rho}+\nabla_{\rho} u_{\lambda}-\frac{2}{d-1} g_{\lambda \rho} \nabla_{\alpha} u^{\alpha}\right)
\end{aligned}
$$

where all coefficients are functions of $\tau$ and $\hat{\mu}$. We have used notations to coincide with the stress tensor and current following from (2.9).

At zeroth derivative order, equation (5.18) requires $\epsilon_{0}, p_{0}, n_{0}$ satisfy the standard thermodynamic relations

$$
\epsilon_{0}+p_{0}=-\frac{\partial p_{0}}{\partial \tau}, \quad n_{0}=\beta \frac{\partial p_{0}}{\partial \hat{\mu}}
$$

with

$$
V_{(0,0)}^{\mu}=p_{0} \beta^{\mu}
$$

In other words, equation (5.18) imposes local first law of thermodynamics.

To examine implications of the first equation of (5.16) we also need to write down the most general form of

$$
W_{0}^{\mu \nu, M N}=W_{0}^{\nu \mu, N M}
$$

with zero derivative. More explicitly we can then write

$$
\begin{aligned}
W_{0}^{\mu \alpha, \nu \beta}= & s_{11} u^{\mu} u^{\nu} u^{\alpha} u^{\beta}+s_{22} \Delta^{\mu \nu} \Delta^{\alpha \beta}-s_{12}\left(u^{\mu} u^{\nu} \Delta^{\alpha \beta}+u^{\alpha} u^{\beta} \Delta^{\mu \nu}\right) \\
& +2 r_{11}\left(u^{\mu} u^{(\alpha} \Delta^{\beta) \nu}+u^{\nu} u^{(\alpha} \Delta^{\beta) \mu}\right)+4 r\left(\Delta^{\alpha(\mu} \Delta^{\nu) \beta}-\frac{1}{d-1} \Delta^{\mu \nu} \Delta^{\alpha \beta}\right) \\
W_{0}^{\mu \alpha, \nu d}= & -s_{13} u^{\mu} u^{\nu} u^{\alpha}+s_{23} \Delta^{\mu \nu} u^{\alpha}+2 r_{12} u^{(\mu} \Delta^{\nu) \alpha}, \quad W_{0}^{\mu \alpha, d \nu}=W_{0}^{\alpha \mu, \nu d} \\
W_{0}^{\mu \nu, d d}= & s_{33} u^{\mu} u^{\nu}+r_{22} \Delta^{\mu \nu} .
\end{aligned}
$$


Again we have chosen notations to be consistent with fluid spacetime action (2.19). It is also convenient to decompose $\Phi_{r \mu M}$ in terms of transverse traceless tensors, transverse vectors, and scalars with respect to $u^{\mu}$

$$
\begin{aligned}
& \left(\Delta_{\mu}{ }^{\lambda} \Delta_{\nu}{ }^{\rho}-\frac{\Delta_{\mu \nu}}{d-1} \Delta^{\lambda \rho}\right) \Phi_{r \mu \nu}=\beta \sigma_{\mu \nu}, \quad \Delta_{\mu}{ }^{\nu} u^{\lambda} \Phi_{r \nu \lambda}=\beta v_{1 \mu}, \quad u^{\mu} u^{\nu} \Phi_{r \mu \nu}=-2 \beta \partial \tau \\
& \Delta^{\mu \nu} \Phi_{r \mu \nu}=2 \beta \theta, \quad \Delta_{\mu}{ }^{\nu} \Phi_{r \nu d}=2 \beta v_{2 \mu}, \quad u^{\mu} \Phi_{r \mu d}=2 \partial \hat{\mu}
\end{aligned}
$$

where

$$
v_{1 \mu}=\partial u_{\mu}-\Delta_{\mu}{ }^{\nu} \partial_{\nu} \tau, \quad v_{2 \mu}=\beta^{-1} \Delta_{\mu}{ }^{\nu} \nabla_{\nu} \hat{\mu}-u^{\nu} F_{\mu \nu} .
$$

Now plugging (5.32)-(5.37) into the first equation of (5.16) and comparing with the first order part of (5.21), we again recover $(2.27),(2.30)$, and (2.31)-(2.33). Note that the Onsager relations follow from (5.31).

\subsection{Entropy current}

Using (5.30), to first order in derivative expansion the entropy current (5.20) has the form

$$
S^{\mu}=p_{0} \beta^{\mu}-T^{\mu \nu} \beta_{\nu}-J^{\mu} \hat{\mu}
$$

which recovers the standard result. Taking the divergence of the above expression, using equations of motion (3.38) and (5.16), (5.18) we find that

$$
\nabla_{\mu} S^{\mu}=\frac{1}{4} W_{0}^{\mu \nu, M N} \Phi_{r \mu M} \Phi_{r \nu N} \equiv Q_{2}
$$

which agrees with the general result of [28]. $\quad Q_{2} \geq 0$ follows from (1.12). Now using (5.32)-(5.37) we can write the right hand side of the above equation as

$$
\begin{aligned}
Q_{2}= & \beta^{2}\left[r \sigma^{\mu \nu} \sigma_{\mu \nu}+r_{11} v_{1}^{2}+r_{22} v_{2}^{2}+2 r_{12} v_{1} \cdot v_{2}\right. \\
& \left.+s_{11}(\partial \tau)^{2}+s_{22} \theta^{2}+\frac{s_{33}}{\beta^{2}}(\partial \hat{\mu})^{2}+2 s_{12} \theta \partial \tau+\frac{2 s_{23}}{\beta} \theta \partial \hat{\mu}+\frac{2 s_{13}}{\beta} \partial \tau \partial \hat{\mu}\right] .
\end{aligned}
$$

Using the ideal fluid equations of motion

$$
v_{1 \mu}=-\frac{n_{0}}{\epsilon_{0}+p_{0}} v_{2 \mu}, \quad \partial \tau=\left(\frac{\partial p_{0}}{\partial \varepsilon_{0}}\right)_{n_{0}} \theta, \quad \frac{1}{\beta} \partial \hat{\mu}=-\left(\frac{\partial p_{0}}{\partial n_{0}}\right)_{\varepsilon_{0}} \theta
$$

in $Q_{2}$ then we find

$$
\nabla_{\mu} S^{\mu}=\frac{\beta}{2} \eta \sigma^{\mu \nu} \sigma_{\mu \nu}+\beta \zeta \theta^{2}+\sigma \beta v_{2}^{2},
$$

where

$$
\begin{aligned}
\sigma & =\frac{\beta}{\left(\varepsilon_{0}+p_{0}\right)^{2}}\left(r_{11} n_{0}^{2}-2 r_{12} n_{0}\left(\epsilon_{0}+p_{0}\right)+r_{22}\left(\epsilon_{0}+p_{0}\right)^{2}\right) \\
\zeta & =\beta\left(s_{11}\left(\partial_{\varepsilon} p_{0}\right)^{2}+s_{22}+s_{33}\left(\partial_{n} p_{0}\right)^{2}+2 s_{12} \partial_{\varepsilon} p_{0}-2 s_{13} \partial_{\varepsilon} p_{0} \partial_{n} p_{0}-2 s_{23} \partial_{n} p_{0}\right)
\end{aligned}
$$


with $\partial_{\varepsilon} p_{0} \equiv\left(\frac{\partial p_{0}}{\partial \varepsilon_{0}}\right)_{n_{0}}$ and $\partial_{n} p_{0} \equiv\left(\frac{\partial p_{0}}{\partial n_{0}}\right)_{\varepsilon_{0}}$. In the first term of (5.42) we have used the first equation of (2.31), and $\sigma, \zeta$ are precisely the expressions for conductivity and bulk viscosity identified in [1], see equations (5.113) and (5.114) there. ${ }^{13}$ We have thus recovered the standard form for the divergence of the entropy current.

\section{$6 \quad$ Frame choices from field redefinitions}

Going to higher orders in derivative expansion the analysis becomes very tedious. Even at the order of $T_{1}^{\mu M}$ and $W_{0}^{\mu \nu, M N}$, the Lagrangian is already pretty long. In this section we show that the Lagrangian can be greatly simplified by taking advantage of field redefinitions.

\subsection{General discussion of field redefinitions}

Let us write the Lagrangian in the form

$$
\mathcal{L}=\mathcal{L}_{1}+\mathcal{L}_{r}
$$

where $\mathcal{L}_{1}$ given by (5.14), $T_{0}^{\mu \nu}=\varepsilon_{0} u^{\mu} u^{\nu}+p_{0} \Delta^{\mu \nu}$ and $J_{0}^{\mu}=n_{0} u^{\mu}$ are the ideal stress tensor and current, and $\mathcal{L}_{r}$ denotes the rest of the Lagrangian. Note that the separation in (6.1) is natural as $\mathcal{L}_{1}$ and $\mathcal{L}_{r}$ are invariant separately under the dynamical KMS condition, i.e.

$$
\tilde{\mathcal{L}}_{1}-\mathcal{L}_{1}=i \nabla_{\mu} V_{(0,0)}^{\mu}, \quad \tilde{\mathcal{L}}_{r}-\mathcal{L}_{r}=\partial_{\mu} V_{r}^{\mu}, \quad V_{r}^{\mu}=V^{\mu}-i V_{(0,0)}^{\mu}
$$

We will denote the equations of motion of $\mathcal{L}_{1}$ as

$$
E_{\mu}=0, \quad E_{1}=0, \quad E_{2}=0,
$$

where

$$
E_{\mu} \equiv\left(\epsilon_{0}+p_{0}\right) v_{1 \mu}+n_{0} v_{2 \mu}, \quad E_{1} \equiv-\partial \tau+\left(\frac{\partial p_{0}}{\partial \varepsilon_{0}}\right)_{n_{0}} \theta, \quad E_{2} \equiv \frac{1}{\beta} \partial \hat{\mu}+\left(\frac{\partial p_{0}}{\partial n_{0}}\right)_{\varepsilon_{0}} \theta
$$

Note that $E_{\mu}$ is the transverse part of the first equation of (3.38) while $E_{1,2}$ are related to the longitudinal part of the first equation and the second equation by a linear transform. ${ }^{14}$

Let us first consider field redefinitions of the $a$-fields

$$
X_{a}^{\mu} \rightarrow X_{a}^{\mu}+\delta X_{a}^{\mu}, \quad \varphi_{a} \rightarrow \varphi_{a}+\delta \varphi_{a}
$$

under which

$$
G_{a \mu \nu} \rightarrow G_{a \mu \nu}+2 \nabla_{(\mu} \delta X_{a \nu)}, \quad G_{a \mu d} \rightarrow G_{a \mu d}+2 \partial_{\mu} \delta \varphi_{a}+2\left(\partial_{\mu}\left(\delta X_{a}^{\nu} A_{\nu}\right)-F_{\mu \nu} \delta X_{a}^{\nu}\right)
$$

${ }^{13}$ To compare (5.44) with (5.115) of [1] note that $M_{1,2,3}$ defined there are related to $\partial_{\varepsilon} p_{0}$ and $\partial_{n} p_{0}$ as

$$
\left(\frac{\partial p_{0}}{\partial \varepsilon_{0}}\right)_{n_{0}}=-\frac{M_{1}}{M_{2}}, \quad\left(\frac{\partial p_{0}}{\partial n_{0}}\right)_{\varepsilon_{0}}=-\frac{M_{3}}{M_{2}}
$$

${ }^{14}$ More explicitly, $\nabla_{\mu} J^{\mu}=-\partial_{\tau} n_{0} E_{1}+\beta \partial_{\hat{\mu}} n_{0} E_{2}$ and $u_{\nu} \nabla_{\mu} T^{\mu \nu}=\partial_{\tau} \epsilon_{0} E_{1}-\beta \partial_{\hat{\mu}} \epsilon_{0} E_{2}$. 
$\delta X_{a}^{\mu}$ and $\delta \varphi_{a}$ can be expanded in terms of number of derivatives and $a$-fields, e.g.

$$
\delta X_{a \mu}=\delta X_{a \mu}^{(1)}+i \delta X_{a \mu}^{(2)}+\delta X_{a \mu}^{(3)}+\cdots
$$

where $\delta X_{a \mu}^{(n)}$ contains $n$ factors of $G_{a \mu M}$ and all terms should start at zero derivative order. Similarly with $\delta \varphi_{a}$. The corresponding $\delta G_{a \mu M}^{(n)}$ have similar expansions and all terms start at first derivative order. Under $(6.5), \mathcal{L}_{1}$ is invariant and

$$
\begin{aligned}
& \mathcal{L}_{r} \rightarrow \mathcal{L}_{r}+\delta_{a} \mathcal{L}_{1}, \quad \delta_{a} \mathcal{L}_{1}=\nabla_{\mu} N_{a}^{\mu}-E_{\mu} \delta X_{a}^{\mu}-E_{1} \delta \lambda_{a 1}-E_{2} \delta \lambda_{a 2} \equiv \nabla_{\mu} N_{a}^{\mu}-E_{\alpha} \delta X_{a}^{\alpha} \\
& E_{\alpha} \equiv\left(E_{\mu}, E_{1}, E_{2}\right), \quad \delta X_{a}^{\alpha} \equiv\left(\delta X_{a}^{\mu}, \delta \lambda_{a 1}, \delta \lambda_{a 2}\right)
\end{aligned}
$$

where $\delta_{a} \mathcal{L}_{r}$ has been reabsorbed into $\mathcal{L}_{r}, N_{a}^{\mu}=T_{0}^{\mu \nu} \delta X_{a}^{\mu}+J_{0}^{\mu}\left(\delta \varphi_{a}+\delta X_{a}^{\nu} A_{\nu}\right), \lambda_{a 1}, \lambda_{a 2}$ are linear combinations of $u_{\mu} \delta X_{a}^{\mu}$ and $\delta \varphi_{a}+\delta X_{a}^{\nu} A_{\nu},{ }^{15}$ and $E_{\mu}, E_{1,2}$ were defined in (6.4). In section 6.2 we will show how one can use (6.8) to set to zero terms in $\mathcal{L}_{r}$ proportional to zeroth order equations of motion (6.4), or proportional to their derivatives.

Let us now turn to field redefinitions of $r$-fields. Since the full Lagrangian depends on the $r$-type dynamical fields only through $u^{\mu}, \beta(x)$ and $\mu$, we can in fact consider nonlocal changes of $r$-variables $\sigma^{i}, \varphi$ as far as the corresponding changes in $u^{\mu}, \mu$ are local. Consider field transformations $\sigma^{i} \rightarrow \sigma^{i}+\delta \sigma^{i}, \tau \rightarrow \tau+\delta \tau, \varphi \rightarrow \varphi+\delta \varphi$, with

$$
\delta \sigma^{i}=\partial_{\mu} \sigma^{i} \int d \sigma^{0} b \delta u^{\mu}, \quad \delta \tau=\frac{1}{\beta} \delta \beta, \quad \delta \varphi=\int d \sigma^{0} b \delta \mu
$$

which result in

$$
u^{\mu} \rightarrow u^{\mu}+\delta u^{\mu}, \quad \beta \rightarrow \beta+\delta \beta, \quad \mu \rightarrow \mu+\delta \mu .
$$

$\delta u^{\mu}, \delta \beta, \delta \mu$ can be expanded in terms of number of derivatives and $a$-fields, e.g.

$$
\delta u^{\mu}=\delta u_{0}^{\mu}+i \delta u_{1}^{\mu}+\delta u_{2}^{\mu}+\cdots
$$

where $\delta u_{n}^{\mu}$ contains $n$ factors of $G_{a \mu M} . \delta u_{0}^{\mu}$ starts at first derivatives while $\delta u_{k}^{\mu}$ with $k>1$ start at zeroth derivatives. Similarly with $\delta \beta$ and $\delta \mu$. Under $(6.12), \mathcal{L}_{1}$ is invariant and

$$
\begin{aligned}
\mathcal{L}_{r} & \rightarrow \mathcal{L}_{r}+\delta_{r} \mathcal{L}_{1}, & \delta_{r} \mathcal{L}_{1} & =E_{a \mu} \delta u^{\mu}+E_{a 1} \delta \epsilon_{0}+E_{a 2} \delta n_{0} \equiv E_{a \alpha} \delta u^{\alpha}, \\
E_{a \alpha} & \equiv\left(E_{a \mu}, E_{a 1}, E_{a 1}\right) & \delta u^{\alpha} & =\left(\delta u^{\mu}, \delta \epsilon_{0}, \delta n_{0}\right),
\end{aligned}
$$

where $\delta \epsilon_{0}=\delta \beta \partial_{\beta} \varepsilon_{0}+\delta \mu \partial_{\mu} \varepsilon_{0}, \delta n_{0}=\delta \beta \partial_{\beta} n_{0}+\delta \mu \partial_{\mu} n_{0}$, and

$$
\begin{aligned}
& E_{a \mu}=\left(\left(\varepsilon_{0}+p_{0}\right) u^{\beta} G_{a \alpha \beta}+n_{0} C_{a \alpha}\right) \Delta_{\mu}^{\alpha}, \\
& E_{a 1}=\frac{1}{2} u^{\mu} u^{\nu} G_{a \mu \nu}+\frac{1}{2}\left(\frac{\partial p_{0}}{\partial \varepsilon_{0}}\right)_{n_{0}} \Delta^{\mu \nu} G_{a \mu \nu}, \quad E_{a 2}=u^{\mu} C_{a \mu}+\frac{1}{2}\left(\frac{\partial p_{0}}{\partial n_{0}}\right)_{\varepsilon_{0}} \Delta^{\mu \nu} G_{a \mu \nu} .
\end{aligned}
$$

We can use (6.14) to make frame changes.

Below we give some general discussion how we can use (6.8) and (6.14) to simplify the action and the imposing of dynamical KMS condition.

\footnotetext{
${ }^{15}$ More explicitly,

$\delta \lambda_{a 1}=-\partial_{\tau} \varepsilon_{0} u^{\alpha} \delta X_{a \alpha}-\partial_{\tau} n_{0}\left(\delta \varphi_{a}+\delta X_{a}^{\nu} A_{\nu}\right), \quad \delta \lambda_{a 2}=\beta \partial_{\hat{\mu}} \varepsilon_{0} u^{\alpha} \delta X_{a \alpha}+\beta \partial_{\hat{\mu}} n_{0}\left(\delta \varphi_{a}+\delta X_{a}^{\nu} A_{\nu}\right)$.
} 


\subsection{Landau frame and generalized Landau frame}

Let us first consider the $O(a)$ Lagrangian $\mathcal{L}^{(1)}=\frac{1}{2} T^{\mu M} G_{a \mu M}$ which can be written as

$$
\mathcal{L}^{(1)}=\frac{1}{2}\left(\varepsilon u^{\mu} u^{\nu}+p \Delta^{\mu \nu}+2 u^{(\mu} q^{\nu)}+\Sigma^{\mu \nu}\right) G_{a \mu \nu}+\left(n u^{\mu}+j^{\mu}\right) C_{a \mu}+\nabla_{\mu} H_{a 0}^{\mu}
$$

where

$$
u_{\mu} \Sigma^{\mu \nu}=u_{\mu} q^{\mu}=u_{\mu} j^{\mu}=0, \quad \Delta_{\mu \nu} \Sigma^{\mu \nu}=0,
$$

and $H_{a 0}^{\mu}$ is an $O(a)$ local expression of fields, i.e. it is linear in $G_{a \mu M}$, and may contain derivatives acting on it, as well as on $u^{\mu}, \tau$ and $\mu$. Using (6.17) we can further write $\mathcal{L}^{(1)}$ as

$$
\mathcal{L}^{(1)}=\mathcal{L}_{\text {Lan }}^{(1)}+Q^{\alpha} E_{a \alpha}+\nabla_{\mu} H_{a 0}^{\mu}, \quad Q^{\alpha}=\left(\frac{1}{\varepsilon_{0}+p_{0}} q^{\mu}, \varepsilon, n\right)
$$

where

$$
\mathcal{L}_{\mathrm{Lan}}^{(1)}=\frac{1}{2} T_{\mathrm{Lan}}^{\mu M} G_{a \mu M}=\frac{1}{2} \Theta_{0} \Delta^{\mu \nu} G_{a \mu \nu}+\mathcal{J}_{0}^{\mu} \Delta_{\mu}^{\nu} C_{a \nu}+\frac{1}{2} \Sigma^{\mu \nu} G_{a \mu \nu}
$$

and

$$
\Theta_{0}=p-\varepsilon \partial_{\varepsilon} p_{0}-n \partial_{n} \epsilon_{0}, \quad \mathcal{J}_{0}^{\mu}=j^{\mu}-\frac{n_{0}}{\varepsilon_{0}+p_{0}} q^{\mu} .
$$

We can further isolate parts of $\Theta_{0}, \mathcal{J}_{0}^{\mu}, \Sigma^{\mu \nu}$ which are proportional to ideal fluid equations of motion $E_{\alpha}$, or to their derivatives. Then (6.19) can be written as

$$
\mathcal{L}^{(1)}=\mathcal{L}_{\mathrm{Lan}}^{(1)}+Q^{\alpha} E_{a \alpha}+E_{\alpha} K_{a}^{\alpha}+\nabla_{\mu} H_{a}^{\mu}
$$

where $K_{a}^{\alpha}$ is an $O(a)$ local expression of fields, $H_{a}^{\mu}$ is an $O(a)$ local expression of $a$ - and $r$-type fields, and now $\Theta_{0}, \mathcal{J}_{0}^{\mu}, \Sigma^{\mu \nu}$ in $\mathcal{L}_{\text {Lan }}^{(1)}$ only contain tensors which are not related by ideal equations of motion. Terms in $\mathcal{L}^{(1)}$ that are proportional to derivatives acting on the zeroth order equations have been incorporated in the third term of (6.22) upon integrating them by parts. The total derivatives generated in this step have been incorporated in the last term of (6.22), together with $H_{a 0}^{\mu}{ }^{16}$

Below the Landau frame Lagrangian refers to this minimal form. By choosing field redefinitions

$$
\delta u^{\alpha}=-Q^{\alpha}, \quad \delta X_{a}^{\mu}=-K_{a}^{\mu}
$$

from (6.8) and (6.14) we then obtain $\mathcal{L}^{(1)}=\mathcal{L}_{\text {Lan }}^{(1)}+\nabla_{\mu} H_{a}^{\mu}$, where $N_{a}^{\mu}$ that appears from doing $a$-field redefinitions (6.8) has been absorbed in $H_{a}^{\mu}$.

We can generalize the above discussion to all orders in the $a$-field expansion. Since $\mathcal{L}$ contains at least one factor of $G_{a \mu M}$, we can always separate out such a factor and decompose the coefficient of it in terms of tensors parallel and transverse to $u^{\mu}$, i.e.

$$
\mathcal{L}=\frac{1}{2}\left(\mathcal{E} u^{\mu} u^{\nu}+\mathcal{P} \Delta^{\mu \nu}+2 u^{(\mu} \mathcal{Q}^{\nu)}+\mathcal{S}^{\mu \nu}\right) G_{a \mu \nu}+\left(\mathcal{N} u^{\mu}+\mathcal{I}^{\mu}\right) C_{a \mu}+\nabla_{\mu} \mathcal{H}_{a 0}^{\mu},
$$

where

$$
u_{\mu} \mathcal{S}^{\mu \nu}=u_{\mu} \mathcal{Q}^{\mu}=u_{\mu} \mathcal{I}^{\mu}=0, \quad \Delta_{\mu \nu} \mathcal{S}^{\mu \nu}=0,
$$

\footnotetext{
${ }^{16}$ For example if $\mathcal{L}_{r}$ contains a term of the form $A^{\nu}\left(\nabla^{\mu} E_{1}\right) G_{a \mu \nu}$ we rewrite it as $-E_{1} \nabla^{\mu}\left(A^{\nu} G_{a \mu \nu}\right)+$ $\nabla^{\mu}\left(E_{1} A^{\nu} G_{a \mu \nu}\right)$. The first term contributes to $E_{\alpha} K_{a}^{\alpha}$ and the second term contributes to $H_{a}^{\mu}$.
} 
and $\mathcal{H}_{a 0}^{\mu}$ is a local expression of $a$ - and $r$-type fields which is at least $O(a)$. Note $\mathcal{E}, \mathcal{P}, \mathcal{Q}^{\mu}, \mathcal{S}^{\mu \nu}$ and $\mathcal{N}, \mathcal{I}^{\mu}$ include terms to all orders in the $a$-field expansion.

Similarly as in the earlier discussion we can further write (6.24) as

$$
\mathcal{L}=\mathcal{L}_{\mathrm{Lan}}+\mathcal{Q}^{\alpha} E_{a \alpha}+E_{\alpha} \mathcal{K}_{a}^{\alpha}+\nabla_{\mu} \mathcal{H}_{a}^{\mu}, \quad \mathcal{Q}^{\alpha}=\left(\frac{1}{\varepsilon_{0}+p_{0}} \mathcal{Q}^{\mu}, \mathcal{E}, \mathcal{N}\right)
$$

where again $\mathcal{H}_{a}^{\mu}$ includes $\mathcal{H}_{a 0}^{\mu}$ as well as the total derivatives that come from integrating by parts terms that contribute to $E_{\alpha} \mathcal{K}_{a}^{\alpha}$ above, and $\mathcal{L}_{\text {Lan }}$ has the form

$$
\mathcal{L}_{\mathrm{Lan}}=\frac{1}{2} \Theta \Delta^{\mu \nu} G_{a \mu \nu}+\mathcal{J}^{\mu} C_{a \mu}+\frac{1}{2} \mathcal{S}^{\mu \nu} \Delta_{\mu}{ }^{\alpha} \Delta_{\nu}{ }^{\beta} G_{a \alpha \beta}
$$

with

$$
\Theta=\mathcal{P}-\mathcal{E} \partial_{\varepsilon} p_{0}-\mathcal{N} \partial_{n} \epsilon_{0}, \quad \mathcal{J}=\mathcal{I}^{\mu}-\frac{n_{0}}{\varepsilon_{0}+p_{0}} \mathcal{Q}^{\mu}
$$

In (6.26) we again have separated possible terms which proportional to ideal fluid equations of motion or to their derivatives. Note that $\Theta_{0}, \mathcal{J}_{0}^{\mu}, \Sigma^{\mu}$ correspond respectively to the lowest order terms of $\Theta, \mathcal{J}^{\mu}, \mathcal{S}^{\mu}$ in the $a$-field expansion. By choosing $\delta u^{\alpha}, \delta X_{a}^{\alpha}$ we can then set

$$
\mathcal{L}=\mathcal{L}_{\mathrm{Lan}}+\nabla_{\mu} \mathcal{H}_{a}^{\mu}
$$

where again we absorbed $N_{a}^{\mu}$ in $\mathcal{H}_{a}^{\mu}$. We will refer to (6.27) as in the generalized Landau frame.

There is no unique way to write the Lagrangian in the form (6.24) since for a cross term of $G_{a \mu \nu}$ and $C_{a \mu}$ one can consider it to be either proportional to $G_{a \mu \nu}$ or to $C_{a \mu}$. As a result the generalized Landau frame Lagrangian (6.27) is also not unique. Equivalently, from (6.26) and (6.27), we see that $\mathcal{L}_{r}$ is invariant under

$$
\begin{array}{ll}
\mathcal{J}^{\mu} \rightarrow \mathcal{J}^{\mu}+\Lambda_{v} E_{a}^{\mu}, & \Theta \rightarrow \Theta+\Lambda_{1} E_{a 1}+\Lambda_{2} E_{a 2} \\
\mathcal{Q}^{\mu} \rightarrow \mathcal{Q}^{\mu}-\left(\epsilon_{0}+p_{0}\right) \Lambda_{v} C_{a}^{\mu}, & \mathcal{E} \rightarrow \mathcal{E}-\frac{1}{2} \Lambda_{1} \Delta^{\mu \nu} G_{a \mu \nu}, \quad \mathcal{N} \rightarrow \mathcal{N}-\frac{1}{2} \Lambda_{2} \Delta^{\mu \nu} G_{a \mu \nu}
\end{array}
$$

where $\Lambda_{v}, \Lambda_{1,2}$ are some arbitrary scalar functions (which again has an expansion in $a$ fields). One could take advantage of the freedom of (6.30) to make further simplifications. We will now illustrate this using an explicit example.

\subsection{First order action in the generalized Landau frame}

To illustrate the general discussion above more concretely, let us consider $\mathcal{L}_{2}$ of (5.14), which we copy here for convenience

$$
\mathcal{L}_{2}=\frac{1}{2} T_{1}^{\mu M} G_{a \mu M}+\frac{i}{4} W_{0}^{\mu \nu, M N} G_{a \mu M} G_{a \nu N}
$$

$T_{1}^{\mu M}$ is given by the first derivative part of (5.21), $W_{0}^{\mu \nu, M N}$ given by (5.32)-(5.34), and their coefficients satisfy (2.27), (2.30), and (2.31)-(2.33). Writing (6.32) in the form of (6.24) 
we have (up to the freedom mentioned at the end of last subsection)

$$
\begin{aligned}
\mathcal{E} & =h_{\varepsilon}+\frac{i}{2}\left(s_{11} u^{\lambda} u^{\rho}-s_{12} \Delta^{\lambda \rho}\right) G_{a \lambda \rho}-i s_{13} u^{\lambda} C_{a \lambda}, \\
\mathcal{P} & \left.=h_{p}+\frac{i}{2}\left(s_{22} \Delta^{\lambda \rho}-s_{12} u^{\lambda} u^{\rho}\right)\right) G_{a \lambda \rho}+i s_{23} u^{\lambda} C_{a \lambda} \\
\mathcal{Q}^{\mu} & =q^{\mu}+i r_{11} u^{\lambda} \Delta^{\rho \mu} G_{a \lambda \rho}+i r_{12} \Delta^{\lambda \mu} C_{a \lambda} \\
\mathcal{S}^{\mu \nu} & =-\eta \sigma^{\mu \nu}+2 i r G_{a}^{<\mu \nu>}, \quad \mathcal{N}=h_{n}+\frac{i}{2}\left(-s_{13} u^{\lambda} u^{\rho}+s_{12} \Delta^{\lambda \rho}\right) G_{a \lambda \rho}+i s_{33} u^{\lambda} C_{a \lambda} \\
\mathcal{I}^{\mu} & =j^{\mu}+i r_{12} u^{\lambda} \Delta^{\rho \mu} G_{a \lambda \rho}+i r_{22} \Delta^{\mu \lambda} C_{a \lambda}
\end{aligned}
$$

In the tensor sector we then find

$$
\mathcal{S}^{\mu \nu}=\eta\left(i \beta^{-1} \Delta^{\alpha \mu} \Delta^{\beta \nu} G_{a \alpha \beta}-\sigma^{\mu \nu}\right)=i \beta^{-1} \eta \Delta^{\alpha \mu} \Delta^{\beta \nu} \tilde{G}_{a \alpha \beta}(-x),
$$

where in the above we used the first of (2.31) and (4.29) (see also (5.35)).

In the vector sector, using the shift (6.30) we can choose $\mathcal{J}^{\mu}$ so that its order $O(a)$ term is proportional to $C_{a \mu}$. We then find that $\mathcal{J}^{\mu}$ can be written as

$$
\mathcal{J}^{\mu}=\left(\lambda_{12}+\frac{n_{0}}{\varepsilon_{0}+p_{0}} \lambda_{1}\right) v_{1}^{\mu}-\left(\lambda_{2}+\frac{n_{0}}{\varepsilon_{0}+p_{0}} \lambda_{12}\right) v_{2}^{\mu}+i \beta^{-1} \sigma \Delta^{\mu \alpha} C_{a \alpha}
$$

where we used (2.27), the first of (2.30) and (5.43). Now using ideal equations of motion (6.4) to eliminate $v_{1 \mu}$ we can write the above equation further as

$$
\mathcal{J}^{\mu}=\sigma\left(i \beta^{-1} \Delta^{\mu \alpha} C_{a \alpha}-v_{2}^{\mu}\right)=i \beta^{-1} \sigma \Delta^{\mu \alpha} \tilde{C}_{a \alpha}(-x) \text {. }
$$

In the scalar sector using the shift in (6.30) we can choose the $O(a)$ term of $\Theta$ to be proportional to $\Delta^{\mu \nu} G_{a \mu \nu}$, resulting in

$$
\begin{aligned}
\Theta= & \left(f_{21}-\partial_{\varepsilon} p_{0} f_{11}-\partial_{n} p_{0} f_{31}\right) \partial \tau-\left(f_{22}+\partial_{\varepsilon} p_{0} f_{12}+\partial_{n} p_{0} f_{32}\right) \theta \\
& +\left(f_{23}-\partial_{\varepsilon} p_{0} f_{12}+\partial_{n} p_{0} f_{33}\right) \beta^{-1} \partial \hat{\mu}+\frac{i}{2} \beta^{-1} \zeta \Delta^{\mu \nu} G_{a \mu \nu},
\end{aligned}
$$

where we used (5.44). Further using equations of motion we then find

$$
\Theta=\zeta\left(\frac{i}{2} \beta^{-1} \Delta^{\mu \nu} G_{a \mu \nu}-\theta\right)=\frac{i}{2} \beta^{-1} \zeta \Delta^{\mu \nu} \tilde{G}_{a \mu \nu}(-x),
$$

where to write the $O\left(a^{0}\right)$ part of $\Theta$ we used (2.30) and (2.32)-(2.33).

Finally collecting the above results together we find a remarkably simple expression

$$
\mathcal{L}_{2}=\mathcal{L}_{\mathrm{Lan}}=\frac{i}{4} \beta^{-1} \zeta \Delta^{\mu \nu} \tilde{G}_{a \mu \nu} \Delta^{\alpha \beta} G_{a \alpha \beta}+i \beta^{-1} \sigma \Delta^{\mu \alpha} \tilde{C}_{a \alpha} C_{a \mu}+\frac{i}{2} \beta^{-1} \eta \Delta^{\mu \alpha} \delta^{\nu \beta} \tilde{G}_{a \mu \nu} G_{a \alpha \beta}
$$

where the tilded variables should be evaluated at $-x$. Note that $\mathcal{L}_{\text {Lan }}$ is manifestly dynamical KMS invariant. 


\subsection{Dynamical KMS condition in Landau frame}

Starting with a dynamical KMS invariant action, after field redefinition changes (6.8) and (6.14), the resulting action is in general no longer dynamical KMS invariant. This is fine as the generating functional $W\left[g_{1}, A_{1} ; g_{2}, A_{2}\right]$ of (1.4) should be invariant under such field redefinitions and remains KMS invariant. ${ }^{17}$ Thus it comes as a pleasant surprise that the action involving $T_{1}^{\mu M}$ can be written in a manifest dynamical KMS invariant form in the generalized Landau frame. It would be interesting to explore whether this happens at all odd derivative orders.

In this subsection to prepare for the explicit second order analysis in section 7 we give some general discussion on how the dynamical KMS condition imposes constraints on $T_{\mathrm{Lan}}^{\mu M}$ at even derivative orders.

We start with the Lagrangian obtained from (5.10) for which the dynamical KMS condition is satisfied at $O\left(a^{k}\right)$ for $k \geq 1$ in the $a$-field expansion. One only needs to impose (5.11), which we copy here for convenience

$$
\left(\tilde{\mathcal{L}}_{2 n+1}\right)_{0}=\left(\widetilde{\mathcal{L}^{(1,2 n)}}+\widetilde{\mathcal{L}^{(2,2 n-1)}}+\cdots+\widetilde{\mathcal{L}^{(2 n+1,0)}}\right)_{0}=i \nabla_{\mu} V_{(0,2 n)}^{\mu}, \quad n=1,2, \cdots
$$

Field redefinitions for different $n$ 's can be treated independently, so let us now consider a specific $n$. Consider a field redefinition which takes $\mathcal{L}_{r}^{(1,2 n)}$ to the Landau frame, i.e

$$
\mathcal{L}_{\text {Lan }}^{(1,2 n)}=\mathcal{L}^{(1,2 n)}-E_{a \alpha} \delta u^{\alpha}-\delta X_{a}^{\alpha} E_{\alpha}+\nabla_{\mu} H_{a}^{\mu} .
$$

$\delta u^{\alpha}$ is of $O\left(a^{0}\right)$ and contains $2 n$ derivatives, while $\delta X_{a}^{\alpha}$ is $O(a)$ with $2 n-1$ derivatives. Note none of the other $\mathcal{L}^{(m, 2 n+1-m)}$ in (6.44) is affected by such redefinitions.

Under a dynamical KMS transformation

$$
\widetilde{\mathcal{L}_{\text {Lan }}^{(1,2 n)}}=\widetilde{\mathcal{L}^{(1,2 n)}}-\left(E_{a \alpha}+i \beta E_{\alpha}\right) \delta u^{\alpha}-E_{\alpha}\left(\delta X_{a}^{\alpha}+i \beta \delta Y^{\alpha}\right)+\nabla_{\mu}\left(H_{a}^{\mu}+i Z^{\mu}\right)
$$

where we have used that $\widetilde{\delta u^{\alpha}}(-x)=\delta u^{\alpha}(x), \tilde{E}_{\alpha}(-x)=-E_{\alpha}(x)$, and

$$
\tilde{E}_{a \alpha}(-x)=E_{a \alpha}(x)+i \beta E_{\alpha}(x) .
$$

Since $\delta X_{a}^{\alpha}$ and $H_{a}^{\mu}$ are $O(a)$, under a dynamical KMS transformation they must have the form

$$
\widetilde{\delta X_{a}^{\alpha}}(-x)=-\left(\delta X_{a}^{\alpha}(x)+i \beta \delta Y^{\alpha}(x)\right), \quad \tilde{H}_{a}^{\mu}(-x)=-\left(H_{a}^{\mu}(x)+i Z^{\mu}(x)\right)
$$

for some $\delta Y^{\alpha}$ and $Z^{\mu}$ which are $O\left(a^{0}\right)$, and the overall minus sign on the right hand side is due to that $\delta X_{a}^{\alpha}$ and $H_{a}^{\mu}$ have odd number of derivatives. Note that the tilde operation in (6.46) should be understood in the sense as described below (5.4). One can readily check that (6.46) does not affect the dynamical KMS invariance at $O(a)$, which also follows from that dynamical KMS transformation at order $O(a)$ does not constrain even derivative terms in $\mathcal{L}^{(1)}$.

\footnotetext{
${ }^{17}$ In evaluating the path integral beyond tree-level, one will have to be careful about potential changes in the integration measures due to such field redefinitions.
} 
Now plugging (6.46) into (6.44) we find that

$$
\left(\widetilde{\mathcal{L}_{\text {Lan }}^{(1,2 n)}}\right)_{0}+\sum_{k=2}^{2 n+1}\left(\widetilde{\left.\mathcal{L}_{r}^{(k, 2 n+1}-k\right)}\right)_{0}=i \nabla_{\mu} \bar{V}_{2 n}^{\mu}+i \beta E_{\alpha}\left(\delta u^{\alpha}+\delta Y^{\alpha}\right),
$$

where $\bar{V}_{2 n}^{\mu}=V_{(0,2 n)}^{\mu}+Z^{\mu}$. Note that from (6.20)

$$
\left(\widetilde{\mathcal{L}_{\text {Lan }}^{(1)}}\right)_{0}=i \beta\left(\Theta_{0} \theta+\mathcal{J}_{0}^{\mu} v_{2 \mu}+\frac{1}{2} \Sigma_{0}^{\mu \nu} \sigma_{\mu \nu}\right)
$$

In appendix $\mathrm{C}$ we prove that at any derivative order $m$ and tensor rank $k$, one can always choose a set of basis of the form $\left\{v_{1}^{\mu_{1} \cdots \mu_{k}}, \ldots, v_{p}^{\mu_{1} \cdots \mu_{k}}, w_{1}^{\mu_{1} \cdots \mu_{k}}, \ldots, w_{q}^{\mu_{1} \cdots \mu_{k}}\right\}$, where $v_{i}^{\mu_{1} \cdots \mu_{k}}$ are not related by the ideal fluid equations (6.4), and $w_{s}^{\mu_{1} \cdots \mu_{k}}$ contain at least one factor of (6.4) or their derivatives. For later reference, we call the first ones $v$-type tensors, and the second ones $w$-type tensors.

Now with the definition below (6.22) for the Landau frame, we can then write (6.50) solely in terms of $v$-type tensors, i.e.

$$
\left(\widetilde{\mathcal{L}_{\text {Lan }}^{(1)}}\right)_{0}=\sum_{i} a_{i} v_{i}
$$

where $a_{i}$ are functions of $\tau$ and $\hat{\mu}$. Similar we can write

$$
\begin{aligned}
\sum_{k=2}^{2 n+1}\left(\widetilde{\mathcal{L}_{r}^{(\widehat{k, 2 n+1}-k)}}\right)_{0} & =\sum_{i} b_{i} v_{i}+\sum_{s} c_{i} w_{s} \\
\nabla_{\mu} \bar{V}_{2 n}^{\mu} & =\left(\nabla_{\mu} \bar{V}_{2 n}^{\mu}\right)_{\min }+\sum_{i} d_{s} w_{s}
\end{aligned}
$$

where $\left(\nabla_{\mu} \bar{V}_{2 n}^{\mu}\right)_{\min }$ contains only $v_{i}$ terms. Equation (6.49) can thus be written as

$$
\left(\tilde{\mathcal{L}}_{\text {Lan }}^{(1,2 n)}\right)_{0}+\sum_{i} b_{i} v_{i}=\left(\nabla_{\mu} \bar{V}_{2 n}^{\mu}\right)_{\min }
$$

and

$$
\sum_{s} c_{s} w_{s}=\sum_{s} d_{s} w_{s}+i \beta E_{\alpha}\left(\delta u^{\alpha}+\delta Y^{\alpha}\right)
$$

Equation (6.54) implies when imposing the dynamical KMS condition on $\mathcal{L}_{\text {Lan }}^{(1,2 n)}$ we can set all terms proportional to ideal equations of motion to zero. As we will see in next section, this provides a great simplification.

\subsection{Entropy current in Landau frame}

In this subsection we show that the entropy current in Landau frame satisfies the local second law up to terms that vanish on the ideal fluid equations of motion. To this aim, we need to carefully track the steps in going from (6.49) to the equation of the entropy 
divergence. Recall that eq. (6.49) is a manipulation of the $(2 n+1)$ th derivative order part of (5.7). Summing (6.49) to the lower derivative orders of (5.7) we then find

$$
\sum_{j=0}^{2 n}\left(\widetilde{\mathcal{L}_{\text {Lan }}^{(1, j)}}\right)_{0}+\sum_{j=0}^{2 n} \sum_{k=2}^{j+1}\left(\widetilde{\mathcal{L}_{r}^{(k, j+1-k)}}\right)_{0}=i \nabla_{\mu} \mathcal{V}_{2 n}^{\mu}+i \beta E_{\alpha}\left(\delta u^{\alpha}+\delta Y^{\alpha}\right),
$$

where $\mathcal{V}_{2 n}^{\mu}=\sum_{j=0}^{n} \bar{V}_{2 j}^{\mu}$, and where we assume that the Lagrangian at derivative order lower than $2 n$ is already in Landau frame. Using (6.20) we express the first term in (6.56) in terms of the stress tensor and the charge current,

$$
\frac{1}{2} T_{\mathrm{Lan}}^{\mu M} \Phi_{r \mu M}-i \sum_{j=0}^{2 n} \sum_{k=2}^{j+1}\left(\widetilde{\mathcal{L}_{r}^{(k, j+1-k)}}\right)_{0}=\nabla_{\mu} \mathcal{V}_{2 n}^{\mu}+\beta E_{\alpha}\left(\delta u^{\alpha}+\delta Y^{\alpha}\right),
$$

integrating by parts,

$$
\nabla_{\mu}\left(\mathcal{V}_{2 n}^{\mu}-T_{\mathrm{Lan}}^{\mu M} \beta_{M}\right)=-\nabla_{\mu} T_{\mathrm{Lan}}^{\mu M} \beta_{M}-i \sum_{j=0}^{2 n} \sum_{k=2}^{j+1}\left(\widetilde{\mathcal{L}_{r}^{(k, j+1-k)}}\right)_{0}-\beta E_{\alpha}\left(\delta u^{\alpha}+\delta Y^{\alpha}\right),
$$

where

$$
\nabla_{\mu} T_{\mathrm{Lan}}^{\mu M} \beta_{M}=\left(\nabla_{\mu} T_{\mathrm{Lan}}^{\mu \nu}+J_{\mathrm{Lan}}^{\mu} F_{\mu}{ }^{\nu}\right) \beta_{\nu}+\nabla_{\mu} J_{\mathrm{Lan}}^{\mu} \hat{\mu}, \quad \beta_{M}=\left(\beta_{\mu}, \hat{\mu}\right) .
$$

The $O\left(a^{0}\right)$ part of the exact equations of motion for the Landau frame Lagrangian is

$$
\nabla_{\mu} T_{\text {Lan }}^{\mu \nu}=-J_{\text {Lan }}^{\mu} F_{\mu}{ }^{\nu}, \quad \nabla_{\mu} J_{\text {Lan }}^{\mu}=0,
$$

and imposing the above, eq. (6.58) becomes

$$
\nabla_{\mu}\left(\mathcal{V}_{2 n}^{\mu}-T_{\mathrm{Lan}}^{\mu M} \beta_{M}\right)=-i \sum_{j=0}^{2 n} \sum_{k=2}^{j+1}\left(\widetilde{\mathcal{L}_{r}^{(k, j+1-k)}}\right)_{0}-\beta E_{\alpha}\left(\delta u^{\alpha}+\delta Y^{\alpha}\right)
$$

Eq. (6.61) has the same form as eq. (3.13) in [28], except that in (6.61) we have an additional term on the r.h.s. which is proportional to the ideal fluid equations of motion, whereas the first term was shown in [28] to be always non-negative. This shows that the entropy current $S_{\text {Lan }}^{\mu}$ in Landau frame,

$$
S_{\text {Lan }}^{\mu} \equiv \mathcal{V}^{\mu}-T_{\text {Lan }}^{\mu M} \beta_{M}=\left(p_{0}+\varepsilon_{0}\right) \beta^{\mu}-J_{\text {Lan }}^{\mu} \hat{\mu}+\sum_{j=1}^{n} \bar{V}_{2 j}^{\mu},
$$

is guaranteed to satisfy the local second law at all derivative orders, up to terms that vanish on the ideal equations of motion.

\section{Conformal fluids at second order in derivatives}

In this section we consider the action for a conformal fluid which has some new elements. We will also work out explicitly the corresponding entropy current to second order in derivative expansion using (5.20) and show that it reproduces previous results. 


\subsection{Conformal fluids}

For a conformal system the generating functional (1.1) should in addition be invariant under independent Weyl scalings of two metrics

$$
W\left[g_{1 \mu \nu}, A_{1 \mu}, g_{2 \mu \nu}, A_{2 \mu}\right]=W\left[e^{2 \lambda_{1}} g_{1 \mu \nu}, A_{1 \mu}, e^{2 \lambda_{2}} g_{2 \mu \nu}, A_{2 \mu}\right]
$$

where $\lambda_{s}(x)$ are scalars, and $s=1,2$. For this purpose it is convenient to introduce Weyl invariant fluid spacetime metrics

$$
\hat{h}_{s a b}(\sigma)=\frac{1}{\beta_{s}^{2}} h_{s a b}(\sigma), \quad \beta_{s}=\beta_{0} e^{\tau_{s}(\sigma)}, \quad \tau_{1}=\tau+\frac{1}{2} \tau_{a}, \quad \tau_{2}=\tau-\frac{1}{2} \tau_{a}
$$

where $h_{s a b}$ was defined in (1.5) and $\tau_{a}$ is defined form the determinants of $h_{1 a b}$ and $h_{2 a b}$,

$$
e^{d \tau_{a}}=\sqrt{\operatorname{det}\left(h_{1} h_{2}^{-1}\right)} .
$$

Under Weyl scalings $g_{s \mu \nu}(X) \rightarrow e^{2 \lambda_{s}(X)} g_{s \mu \nu}, \tau_{a}$ transforms as $\tau_{a} \rightarrow \tau_{a}+\lambda_{1}(\sigma)-\lambda_{2}(\sigma)$, and $\hat{h}_{s a b}$ are invariant if $\tau$ transforms as

$$
2 \tau(\sigma) \rightarrow 2 \tau(\sigma)+\lambda_{1}(\sigma)+\lambda_{2}(\sigma), \quad \lambda_{s}(\sigma)=\lambda_{s}(X(\sigma)) .
$$

Equation (7.1) can be satisfied if we require the action to depend only on $\hat{h}_{s a b}$ and $B_{s a}$, i.e.

$$
\text { conformal fluids : } \quad I=I\left[\hat{h}_{1}, B_{1} ; \hat{h}_{2} ; B_{2}\right] .
$$

All the other conditions discussed in the Introduction section remain the same.

One can immediately write down the action either in fluid spacetime or physical spacetime in parallel with earlier discussions using $\hat{h}_{s a b}$ in place of $h_{s a b}$ and dropping any explicit $\tau$-dependence. For illustration we will consider a neutral fluid. In appendix D we discussion the formulation of the action in the fluid spacetime with a finite $\hbar$ (before imposing dynamical KMS symmetry). Here we concentrate on the classical limit.

In the $\hbar \rightarrow 0$ limit using (7.2), one finds

$$
\hat{h}_{1 a b}=\hat{h}_{a b}(\sigma)+\frac{\hbar}{2} \hat{h}_{a b}^{(a)},
$$

with

$$
\begin{aligned}
\hat{h}_{a b}(\sigma) & \equiv \partial_{a} X^{\mu} \partial_{b} X^{\nu} \hat{g}_{\mu \nu}(X), \quad \hat{h}_{a b}^{(a)}=\partial_{a} X^{\mu} \partial_{b} X^{\nu} \hat{G}_{a \mu \nu}(X), \\
\hat{g}_{\mu \nu} & \equiv \beta^{-2}(x) g_{\mu \nu}, \quad \hat{G}_{a \mu \nu} \equiv\left(\beta^{-2}(x) g_{a \mu \nu}+\mathcal{L}_{X_{a}} \hat{g}_{\mu \nu}\right)_{\text {traceless }}=\beta^{-2}(x)\left(G_{a \mu \nu}\right)_{\text {traceless }},
\end{aligned}
$$

where $\beta(x)$ is defined as in (1.3), and the traceless part of a tensor $A$ is defined as

$$
\left(A_{\mu \nu}\right)_{\text {traceless }}=A_{\mu \nu}-\frac{1}{d} A_{\alpha \beta} \hat{g}^{\alpha \beta} \hat{g}_{\mu \nu}
$$

with $\hat{g}^{\mu \nu}$ the inverse of $\hat{g}_{\mu \nu}$. The action in physical space-time will now depend on $\beta^{\mu}, \hat{g}_{\mu \nu}, \hat{G}_{a \mu \nu}$ with

$$
\beta^{\mu}=\frac{1}{\hat{b}} \partial_{0} X^{\mu}, \quad \hat{b}=\sqrt{-\hat{g}_{\mu \nu} \partial_{0} X^{\mu} \partial_{0} X^{\nu}}
$$


For spacetime derivatives we will always use the covariant derivative $\hat{\nabla}_{\mu}$ associated with $\hat{g}_{\mu \nu}$. The dynamical KMS transformations (4.29) now have the form

$$
\beta^{\mu}(x) \rightarrow \beta^{\mu}(-x), \quad \hat{g}_{\mu \nu}(x) \rightarrow \hat{g}_{\mu \nu}(-x), \quad \hat{G}_{a \mu \nu}(-x) \rightarrow \hat{G}_{a \mu \nu}(x)+2\left(\hat{\nabla}_{(\mu} \beta_{\nu)}\right)_{\text {traceless }}(x),
$$

where we use $\hat{g}_{\mu \nu}$ and its inverse to raise and lower indices. In particular, note that in terms of usual velocity field $u^{\mu}$,

$$
\beta^{\mu}=\beta u^{\mu}, \quad \beta_{\mu}=\hat{g}_{\mu \nu} \beta^{\nu}=\beta^{-1}(x) g_{\mu \nu} u^{\nu}, \quad \beta^{\mu} \beta_{\mu}=\hat{g}_{\mu \nu} \beta^{\mu} \beta^{\nu}=-1 .
$$

Now the action can be written as

$$
I_{\text {hydro }}=\int d^{d} x \sqrt{-\hat{g}} \hat{\mathcal{L}}, \quad \hat{\mathcal{L}}=\beta^{d} \mathcal{L}
$$

with

$$
\hat{\mathcal{L}}=\frac{1}{2} \hat{T}^{\mu \nu} \hat{G}_{a \mu \nu}+\frac{i}{4} \hat{W}^{\mu \rho, \nu \sigma} \hat{G}_{a \mu \nu} \hat{G}_{a \rho \sigma}+\frac{1}{8} \hat{Y}^{\mu \rho \alpha, \nu \sigma \beta} \hat{G}_{a \mu \nu} \hat{G}_{a \rho \sigma} \hat{G}_{a \alpha \beta}+\cdots .
$$

where $\hat{T}^{\mu \nu}, \hat{W}^{\mu \nu, \lambda \rho}$ and $\hat{Y}^{\mu \nu \rho, \lambda \sigma \delta}$ are functions of $\beta^{\mu}, \hat{g}_{\mu \nu}$, their derivatives, and derivative operators acting on $\hat{G}_{a \mu M}$. Note that since $\hat{G}_{a \mu \nu}$ is traceless, the trace components $\hat{T}^{\mu \nu} \hat{g}_{\mu \nu}, \hat{X}^{\mu \rho, \nu \sigma} \hat{g}_{\mu \nu}, \ldots$ decouple from the action, and we shall thus take such components to be zero in what follows. From (7.11), these hatted tensors are related to the un-hatted ones through $T^{\mu \nu}=\beta^{-(d+2)}(x) \hat{T}^{\mu \nu}, W^{\mu \rho, \nu \sigma}=\beta^{-(d+4)}(x) \hat{W}^{\mu \rho, \nu \sigma}$ and $Y^{\mu \rho \alpha, \nu \sigma \beta}=\beta^{-(d+6)}(x) \hat{Y}^{\mu \rho \alpha, \nu \sigma \beta}$.

To second order in derivative expansion, the dynamical KMS conditions can be imposed using (5.16)-(5.19). At zeroth and first order the analysis are the same as before and we find

$$
\begin{aligned}
\hat{T}_{0}^{\mu \nu}= & \hat{p}_{0}\left((d-1) \beta^{\mu} \beta^{\nu}+\hat{\Delta}^{\mu \nu}\right) \\
\hat{T}_{1}^{\mu \nu}= & -(d-1) \hat{f}_{22} \hat{\theta} \beta^{\mu} \beta^{\nu}-\hat{f}_{22} \hat{\theta} \hat{\Delta}^{\mu \nu}-2 \hat{\lambda}_{1} \beta^{(\mu} \hat{\partial} \beta^{\nu)}-\hat{\eta} \hat{\sigma}^{\mu \nu} \\
\hat{W}_{0}^{\mu \alpha, \nu \beta}= & (d-1)^{2} \hat{s}_{22} \beta^{\mu} \beta^{\nu} \beta^{\alpha} \beta^{\beta}+\hat{s}_{22} \hat{\Delta}^{\mu \nu} \hat{\Delta}^{\alpha \beta}+(d-1) \hat{s}_{22}\left(\beta^{\mu} \beta^{\nu} \hat{\Delta}^{\alpha \beta}+\beta^{\alpha} \beta^{\beta} \hat{\Delta}^{\mu \nu}\right) \\
& +2 \hat{r}_{11}\left(\beta^{\mu} \beta^{(\alpha} \hat{\Delta}^{\beta) \nu}+\beta^{\nu} \beta^{(\alpha} \hat{\Delta}^{\beta) \mu}\right)+4 \hat{r} \hat{\Delta}^{\alpha<\mu} \hat{\Delta}^{\nu>\beta}
\end{aligned}
$$

where all coefficients are constants,

$$
\hat{\Delta}^{\mu \nu}=\hat{g}^{\mu \nu}+\beta^{\mu} \beta^{\nu}, \quad \hat{\sigma}_{\mu \nu}=2 \hat{\nabla}_{<\mu} \beta_{\nu>}, \quad \hat{\theta}=\hat{\nabla}_{\mu} \beta^{\mu}, \quad \hat{\partial}=\beta^{\mu} \hat{\nabla}_{\mu},
$$

and $A^{<\mu \nu>}$ denotes the symmetric transverse traceless part of a tensor $A^{\mu \nu}$, i.e.

$$
A^{<\mu \nu>} \equiv A^{(\alpha \beta)} \hat{\Delta}_{\alpha}^{\mu} \hat{\Delta}_{\beta}^{\nu}-\frac{1}{d-1} A^{\alpha \beta} \hat{\Delta}_{\alpha \beta} \hat{\Delta}^{\mu \nu}
$$

Invariance of $\hat{\mathcal{L}}$ under (7.11) gives the following relations among coefficients in (7.16)-(7.18)

$$
\hat{r}=\frac{1}{2} \hat{\eta}, \quad \hat{r}_{11}=\hat{\lambda}_{1}, \quad \hat{s}_{22}=\hat{f}_{22}
$$

which is the conformal limit of (2.31)-(2.33). Interestingly, equations (2.27) and (2.30) are automatically satisfied in the conformal case. 


\subsection{Analysis at second order}

To obtain the explicit form of the entropy current (5.20) to second derivative order, in this subsection we work out the constraint (5.19) for a conformal fluid for which it becomes

$$
\hat{T}_{2}^{\mu \nu} \hat{\nabla}_{\mu} \beta_{\nu}+\frac{1}{2} \hat{Y}_{0}^{\mu \rho \alpha, \nu \sigma \beta} \hat{\nabla}_{\mu} \beta_{\nu} \hat{\nabla}_{\rho} \beta_{\sigma} \hat{\nabla}_{\alpha} \beta_{\beta}=\hat{\nabla}_{\mu} \hat{V}_{(0,2)}^{\mu},
$$

where $\hat{V}_{(0,2)}^{\mu}=\beta^{d}(x) V_{(0,2)}^{\mu}$, and we have used $\nabla_{\mu} V_{(0,2)}^{\mu}=\beta^{-d}(x) \hat{\nabla}_{\mu} \hat{V}_{(0,2)}^{\mu}$. To simplify the analysis we will apply the discussion of section 6.4: we will go to Landau frame and set to zero terms that vanish on the ideal fluid equations of motion. Note in the conformal case the ideal fluid equations $\nabla_{\mu} T_{0}^{\mu \nu}=0$ can be written as

$$
\hat{\partial} \beta_{\mu}=0, \quad \hat{\theta}=0 .
$$

The explicit expression of $\hat{T}_{2}^{\mu \nu}$ in Landau frame is

$$
\hat{T}_{2}^{\mu \nu}=f_{1} \hat{R}^{<\mu \nu>}+f_{2} \hat{\sigma}_{\alpha}^{<\mu} \hat{\sigma}^{\nu>\alpha}+f_{3} \hat{\omega}_{\alpha}^{<\mu} \hat{\omega}^{\nu>\alpha}+f_{4} \hat{\sigma}_{\alpha}^{<\mu} \hat{\omega}^{\nu>\alpha}+f_{5}(\hat{\partial} \hat{\sigma})^{<\mu \nu>},
$$

with

$$
\hat{\omega}^{\mu \nu} \equiv-2 \hat{\Delta}^{\mu \alpha} \hat{\Delta}^{\nu \beta} \hat{\nabla}_{[\alpha} \beta_{\beta]}, \quad(\hat{\partial} \hat{\sigma})^{<\mu \nu>} \equiv \hat{\Delta}^{\mu \alpha} \hat{\Delta}^{\nu \beta} \hat{\partial}\left(\hat{\sigma}_{\alpha \beta}\right)-\frac{1}{d-1} \hat{\Delta}^{\mu \nu} \hat{\Delta}^{\alpha \beta} \hat{\partial}\left(\hat{\sigma}_{\alpha \beta}\right)
$$

where $\hat{R}_{\alpha \beta}$ is the Ricci tensor of $\hat{g}_{\mu \nu}$, and $f_{1}, f_{2}, \ldots$ are constant, and where again we neglected terms that vanish on (7.23), such as $\hat{\theta} \hat{\sigma}^{\mu \nu}$. Similarly after writing down the most general tensor form of $\hat{Y}_{0}^{\mu \rho \alpha, \nu \sigma \beta}$, contracting it with $\hat{\nabla}_{\mu} \beta_{\nu}$, and setting to zero all terms proportional to (7.23), we find for the second term of (7.22) only one term survives

$$
\frac{1}{16} \hat{Y}_{0}^{\mu \rho \alpha, \nu \sigma \beta} \hat{\sigma}_{\mu \nu} \hat{\sigma}_{\rho \sigma} \hat{\sigma}_{\alpha \beta}=\frac{1}{16} h_{1} \hat{\sigma}_{\alpha}^{\mu} \hat{\sigma}^{\nu \alpha} \hat{\sigma}_{\mu \nu}
$$

with $h_{1}$ a constant. Finally, the most general expression for $\hat{V}_{(0,2)}^{\mu}$ is ${ }^{18}$

$$
\hat{V}_{(0,2)}^{\mu}=\left(c_{1} \hat{R}+c_{2} \hat{\sigma}^{2}+c_{3} \hat{\omega}^{2}\right) \beta^{\mu}+v_{1} \hat{\nabla}_{\nu} \hat{\omega}^{\mu \nu}+v_{2}\left(\hat{R}^{\mu \nu}-\frac{1}{2} \hat{g}^{\mu \nu} \hat{R}\right) \beta_{\nu}
$$

where $\omega^{2}=\omega_{\mu \nu} \omega^{\mu \nu}$ and $c_{1}, c_{2}, \ldots$ are constant. In writing down (7.24)-(7.27) we have used the identities of appendix $\mathrm{E}$ which guarantee that these are the most general second order expressions.

Plugging (7.24), (7.26) and (7.27) into (7.22), and using again the identities of appendix E, we find

$$
\begin{gathered}
\frac{1}{4} f_{5} \hat{\partial}\left(\hat{\sigma}^{2}\right)+\frac{1}{2} f_{3} \hat{\omega}_{\alpha}^{\mu} \hat{\omega}^{\nu \alpha} \hat{\sigma}_{\mu \nu}+\left(\frac{1}{2} f_{2}+\frac{1}{16} h_{1}\right) \hat{\sigma}_{\alpha}^{\mu} \hat{\sigma}^{\nu \alpha} \hat{\sigma}_{\mu \nu}+\frac{1}{2} f_{1} \hat{R}^{\mu \nu} \hat{\sigma}_{\mu \nu} \\
=c_{1} \hat{\partial} \hat{R}+c_{2} \hat{\partial}\left(\hat{\sigma}^{2}\right)-2 c_{3} \hat{\omega}^{\mu}{ }_{\alpha} \hat{\omega}^{\nu \alpha} \hat{\sigma}_{\mu \nu}+\frac{1}{2} v_{2} \hat{R}^{\mu \nu} \hat{\sigma}_{\mu \nu},
\end{gathered}
$$

\footnotetext{
${ }^{18}$ Note that below we are identifying $V_{(0,2)}^{\mu}$ with $\bar{V}_{2 n}^{\mu}$ introduced below (6.49).
} 
where in evaluating the divergence $\hat{\nabla}_{\mu} \hat{V}_{(0,2)}^{\mu}$ we again neglected terms that vanish on the ideal fluid equations. Eq. (7.28) gives the relations

$$
c_{1}=0, \quad c_{2}=\frac{1}{4} f_{5}, \quad c_{3}=-\frac{1}{4} f_{3}, \quad v_{2}=f_{1}, \quad f_{2}=-\frac{1}{8} h_{1},
$$

Note that $v_{1}$ does not appear in the above relations as in (7.27) it multiplies a term of zero divergence. ${ }^{19}$ Equation (7.29) gives

$$
V_{(0,2)}^{\mu}=\beta^{-d} \hat{V}_{(0,2)}^{\mu}=\frac{1}{4}\left(f_{5} \hat{\sigma}^{2}-f_{3} \hat{\omega}^{2}\right) \beta^{\mu}+v_{1} \hat{\nabla}_{\nu} \hat{\omega}^{\mu \nu}+f_{1}\left(\hat{R}^{\mu \nu}-\frac{1}{2} \hat{g}^{\mu \nu} \hat{R}\right) \beta_{\nu} .
$$

In the above analysis we did not find the relation

$$
f_{5}+f_{4}-2 f_{2}=0
$$

which was observed as a universal relation in holographic theories dual to Einstein gravity [31]. Equation (7.31) was moreover found to be present in the first order correction in various higher derivative theories [32, 33], but fail non-perturbatively in Gauss-Bonnet coupling $[33,34]$ (which was independently verified at second order in Gauss-Bonnet coupling in [35]). Our conclusion is consistent with the discussion in [33] that such a relation cannot hold universally in hydrodynamics.

\subsection{Entropy current at second order}

From (5.20) the expression for the entropy current at second order, for a conformal neutral fluid, is

$$
S_{2}^{\mu}=V_{(0,2)}^{\mu}-T_{2}^{\mu \nu} \beta_{\nu}=V_{(0,2)}^{\mu}
$$

where the second equality follows from that $T_{2}^{\mu \nu}$ is in Landau frame. The expression $(7.32)$ with $V_{(0,2)}^{\mu}$ given by (7.30) agrees precisely with the expression previously given in $[36,37]$, except that with the method of $[36,37] c_{2}$ was undetermined (see also [38]). Taking the divergence of the total entropy current (5.20), using the equations of motion (3.38), (5.16), (5.18) and (5.19), the third order part of the entropy divergence is

$$
\left(\nabla_{\mu} S^{\mu}\right)_{3}=-\frac{1}{2} T_{2}^{\mu \nu} \Phi_{r \mu \nu}+\nabla_{\mu} V_{(0,2)}^{\mu}=\frac{1}{16} Y_{0}^{\mu \rho \alpha, \nu \sigma \beta} \Phi_{r \mu \nu} \Phi_{r \rho \sigma} \Phi_{r \alpha \beta}
$$

which leads to

$$
\left(\nabla_{\mu} S^{\mu}\right)_{3}=-\frac{1}{2} T^{d-3} f_{2} \sigma_{\alpha}^{\mu} \sigma^{\nu \alpha} \sigma_{\mu \nu}
$$

Note that the right hand side of the above equation does not have a definite sign. This term is subleading in derivative expansion compared with the first term in (5.42). Altogether, up to third order we have

$$
\nabla_{\mu} S^{\mu}=\frac{1}{2} T^{-1} \eta \sigma^{\mu \nu} \sigma_{\mu \nu}-\frac{1}{2} T^{d-3} f_{2} \sigma_{\alpha}^{\mu} \sigma^{\nu \alpha} \sigma_{\mu \nu}
$$

\footnotetext{
${ }^{19}$ This comes from the identity $\left[\hat{\nabla}_{\mu}, \hat{\nabla}_{\nu}\right] \hat{\omega}^{\mu \nu}=2 \hat{R}_{\mu \nu} \hat{\omega}^{\mu \nu}=0$, which holds without imposing the ideal fluid equations.
} 
As shown in [28], the entropy divergence can always be written as a square, up to higher derivative terms. Applying the algorithm constructed in [28] we write (7.35) as

$$
\nabla_{\mu} S^{\mu}=\frac{1}{2} T^{-1} \eta\left(\sigma^{\mu \nu} \sigma_{\mu \nu}-\frac{1}{2} T^{d-2} \frac{f_{2}}{\eta} \sigma_{\alpha}^{\mu} \sigma^{\nu \alpha}\right)^{2}-\frac{1}{8} T^{2 d-5} \frac{f_{2}^{2}}{\eta} \sigma_{\alpha}^{\mu} \sigma_{\beta}^{\alpha} \sigma_{\gamma}^{\beta} \sigma_{\mu}^{\gamma}
$$

the last term is fourth order in derivatives, hence it can be neglected, and we are left with a non-negative divergence.

\section{Conclusions and discussions}

In this paper we further developed the fluctuating hydrodynamics proposed in [1] in a number of directions. We first elucidated the structure of the hydrodynamic action in the classical limit, which enables a transparent formulation of the action in physical spacetime in the presence of arbitrary external fields. It also makes connections to some of the earlier work [2-13] clearer. We then proposed a dynamical KMS symmetry which ensures local equilibrium. The dynamical KMS symmetry is physically equivalent to the previously proposed local KMS condition in the classical limit, but is more convenient to implement and more general. It should be applicable to any states in local equilibrium rather than just thermal density matrix perturbed by external sources. We then discussed making frame choices using field redefinitions, which can be used to significantly simplify the action and the imposition of the dynamical KMS symmetry. We discussed how to go to the Landau frame and generalized Landau frame. Finally we proposed a formulation for a conformal fluid, which requires introducing some new elements. We then worked out the explicit form of the entropy current to second order in derivatives for a neutral conformal fluid using the method of [28]. The result agrees nicely with that in previous literature. We explicitly verified that, while with the existing methods part of the entropy current remains undetermined, our procedure leads to a unique expression solely by using second order transport data.

We pointed out some open issues regarding the formulation of dynamical KMS transformations in the quantum regime. There is a potential ambiguity and at the moment there is no obvious principle to fix it.

There are also other conceptual issues in the quantum regime. For example, let us consider a neutral conformal fluid whose only scale is then the local inverse temperature $\beta$ which provides the UV cutoff for the hydrodynamic effective action. This is, however, also the typical scale of quantum fluctuations. While one can treat quantum effects perturbatively to maintain locality, it appears that there is no separation of scales and thus not clear whether effective field theory approach still makes sense at all in the full quantum regime.

\section{Acknowledgments}

We thank P. Gao, S. Rajagopal and D. T. Son for discussions. Work supported in part by funds provided by the U.S. Department of Energy (D.O.E.) under cooperative research agreement DE-FG0205ER41360. 


\section{A A simple argument}

For this purpose, we first note a general result regarding an on-shell action: suppose an action has a symmetry

$$
I[\chi ; \phi]=I[\tilde{\chi} ; \tilde{\phi}]
$$

where variables with a tilde are related to original variables by some transformation, then

$$
I_{\mathrm{on}-\text { shell }}[\phi]=I_{\mathrm{on}-\operatorname{shell}}[\tilde{\phi}]
$$

To see this, note equation (A.1) implies that

$$
\tilde{\chi}^{\mathrm{cl}}[\phi]=\chi^{\mathrm{cl}}[\tilde{\phi}]
$$

and thus

$$
I_{\mathrm{on}-\operatorname{shell}}[\phi]=I\left[\chi^{\mathrm{cl}}[\phi] ; \phi\right]=I\left[\tilde{\chi}^{\mathrm{cl}}[\phi] ; \tilde{\phi}\right]=I\left[\chi^{\mathrm{cl}}[\tilde{\phi}] ; \tilde{\phi}\right]=I_{\mathrm{on}-\operatorname{shell}}[\tilde{\phi}]
$$

\section{B Absorbing $V^{\mu}$ by total derivatives}

Dynamical KMS invariance requires

$$
\tilde{\mathcal{L}}-\mathcal{L}=\partial_{\mu} V^{\mu}
$$

From $Z_{2}$ nature of the dynamical KMS transformation, acting on (B.1) with another dynamical KMS transform we find

$$
\mathcal{L}-\tilde{\mathcal{L}}=-\partial_{\mu} \tilde{V}^{\mu}
$$

where we used that $\widetilde{\partial_{\mu} V^{\mu}}=-\partial_{\mu} \hat{V}^{\mu}$, where $\tilde{V}^{\mu}$ is the dynamical KMS transform of $V^{\mu}$. We then find

$$
\partial_{\mu} V^{\mu}=\partial_{\mu} \tilde{V}^{\mu}
$$

Splitting $V_{0}^{\mu}$ from $V^{\mu}$, i.e. $V^{\mu}=i V_{0}^{\mu}+V_{a}^{\mu}$, we can write (B.1) as

$$
\tilde{\mathcal{L}}-\mathcal{L}=i \partial_{\mu} V_{0}^{\mu}+\frac{1}{2}\left(\partial_{\mu} V_{a}^{\mu}+\partial_{\mu} \tilde{V}_{a}^{\mu}\right)=i \partial_{\mu} V_{0}^{\mu}+\frac{1}{2}\left(\partial_{\mu} V_{a}^{\mu}-\widetilde{\partial_{\mu} V_{a}^{\mu}}\right) .
$$

Now redefining $\mathcal{L} \rightarrow \mathcal{L}-\frac{1}{2} \partial_{\mu} V_{a}^{\mu}$ we then find that

$$
\tilde{\mathcal{L}}-\mathcal{L}=i \partial_{\mu} V_{0}^{\mu}
$$

i.e. all $V_{k}^{\mu}$ in (5.5) with $k \geq 1$ can be set to zero by shifting $\mathcal{L}$ by a total derivative. 


\section{A special basis}

Consider a generic tensor $T^{\mu_{1} \cdots \mu_{k}}$ which is an $n$th derivative order expression of $u^{\mu}, T$ and $\mu$. This can be expanded in terms of a list of independent order $n$ tensors $u_{(n) 1}^{\mu_{1} \cdots \mu_{k}}, \ldots, u_{(n) m}^{\mu_{1} \cdots \mu_{k}}$, i.e.

$$
T^{\mu_{1} \cdots \mu_{k}}=\sum_{i=1}^{m} c_{i}(\tau, \mu) u_{(n) i}^{\mu_{1} \cdots \mu_{k}},
$$

where $c_{i}(\tau, \mu)$ are functions of $\tau$ and $\mu$, and where (C.1) can be seen as a generalization of (5.23)-(5.27). In the reminder we shall show that the list of the $u_{(n) i}^{\mu_{1} \ldots \mu_{k}}$, s can be rearranged into a list constituted by $v_{(n) 1}^{\mu_{1} \cdots \mu_{k}}, \ldots, v_{(n) p}^{\mu_{1} \cdots \mu_{k}}$ and $w_{(n) 1}^{\mu_{1} \cdots \mu_{k}}, \ldots, w_{(n) q}^{\mu_{1} \cdots \mu_{k}}$, with $p+q=m$, such that the $w_{(n) i}^{\mu_{1} \cdots \mu_{k}}$, s contain at least one factor of $E_{\mu}, E_{1}$ and $E_{2}$ (defined in (6.4)), or one factor of derivatives acting on them, and such that the $v_{(n) i}^{\mu_{1} \cdots \mu_{k}}$ are not related through the ideal equations of motion $E_{\alpha}=0$, i.e. it is not possible to find functions $c_{i}(\tau, \mu)$ and a tensor $w^{\mu_{1} \cdots \mu_{k}}$ such that, for some $v_{(n) j}^{\mu_{1} \cdots \mu_{k}}$,

$$
v_{j}^{\mu_{1} \cdots \mu_{k}}=\sum_{i \neq j}^{p} c_{i} v_{(n) i}^{\mu_{1} \cdots \mu_{k}}+w^{\mu_{1} \cdots \mu_{k}} .
$$

For ease of notation, in the reminder we shall drop the space-time indices $\mu_{1} \cdots \mu_{k}$ and the subscript $(n)$.

First we choose a list of tensors $v_{1}, \ldots, v_{p}$ among $u_{1}, \ldots, u_{m}$ that are independent after imposing $E_{\alpha}=0$, with $p \leq m$. Up to permutations, we can assume that $v_{1}=u_{1}, \ldots$, $v_{p}=u_{p}$. This clearly implies that $u_{p+1}, \ldots, u_{m}$ are generated by $v_{1}, \ldots, v_{p}$ upon using $E_{\alpha}=0$, i.e. there are functions $c_{i j}(\tau, \mu)$ such that

$$
u_{i}=\sum_{j=1}^{p} c_{i j} v_{j}+w_{i-p}, \quad i=p+1, \ldots, m,
$$

where $w_{i}=0$ after setting $E_{\alpha}=0$, for $i=1, \ldots, q$, and $q=m-p$. The latter property implies that $w_{i}$ is proportional to either of $E_{\mu}, E_{1}$ or $E_{2}$, or to derivatives acting on them. Note that the $w_{i}$ 's are independent from each other and from the $v_{i}$ 's. To see this, assume by contradiction that, for some $w_{i}$, with $1 \leq i \leq q$, there are $d_{j}(\tau, \mu)$ and $e_{j}(\tau, \mu)$ such that

$$
w_{i}=\sum_{j=1}^{p} d_{j} v_{j}+\sum_{\substack{j=1 \\ j \neq i}}^{q} e_{j} w_{j},
$$

then, from (C.3),

$$
\begin{aligned}
u_{i+p} & =\sum_{j=1}^{p} c_{i+p, j} v_{j}+\sum_{j=1}^{p} d_{j} v_{j}+\sum_{\substack{j=1 \\
j \neq i}}^{q} e_{j}\left(u_{j+p}-\sum_{k=1}^{p} c_{j+p, k} v_{k}\right) \\
& =\sum_{j=1}^{p}\left(c_{i+p, j}+d_{j}-\sum_{\substack{k=1 \\
k \neq i}}^{q} e_{k} c_{k+p, j}\right) u_{j}+\sum_{\substack{j=1 \\
j \neq i}}^{q} e_{j} u_{j+p},
\end{aligned}
$$

which cannot happen, as $u_{i}$ is independent from $u_{k}$, with $k \neq i$. This concludes the proof. 


\section{Action for conformal fluids in fluid spacetime}

In this appendix, we elaborate more on the explicit form of the conformal charged fluid action in fluid spacetime without taking $\hbar \rightarrow 0$ limit.

The discussion parallels that of a general fluid in section 2.1 so we will only highlight the differences.

Using definition (7.2), we decompose $\hat{h}_{s a b}=e^{-2 \tau_{s}} h_{s a b}$ as

$$
\hat{h}_{s a b} d \sigma^{a} d \sigma^{b}=-E_{s}^{2}\left(d \sigma^{0}-v_{s i} d \sigma^{i}\right)^{2}+\alpha_{s i j} d \sigma^{i} d \sigma^{j},
$$

with

$$
E_{s}=e^{-\tau_{s}} b_{s}, \quad \alpha_{s i j}=e^{-2 \tau_{s}} a_{s i j} .
$$

Instead of (2.2) it is more convenient to decompose $B_{s a}$ as

$$
B_{s a} d \sigma^{a}=\hat{\mu}_{s} E_{s}\left(d \sigma^{0}-v_{s i} d \sigma^{i}\right)+\mathfrak{b}_{s i} d \sigma^{i}
$$

with $\hat{\mu}_{s}=e^{\tau_{s}} \mu_{s}$ and the corresponding symmetric and antisymmetric combinations $\hat{\mu}_{r}=$ $\frac{1}{2}\left(\hat{\mu}_{1}+\hat{\mu}_{2}\right)$ and $\hat{\mu}_{a}=\hat{\mu}_{1}-\hat{\mu}_{2}$. Instead of $(2.3)-(2.5), E_{r, a}, \chi_{a}$ are now defined as

$$
\begin{array}{rlrl}
E_{r} & =\frac{1}{2}\left(E_{1}+E_{2}\right)=\frac{1}{2}\left(e^{-\tau_{1}} b_{1}+e^{-\tau_{2}} b_{2}\right), E_{a} & =\log \left(E_{2}^{-1} E_{1}\right)=-\tau_{a}+\log \left(b_{2}^{-1} b_{1}\right), \\
\alpha_{r i j} & =\frac{1}{2}\left(\alpha_{1 i j}+\alpha_{2 i j}\right), & \chi_{a} & =\frac{1}{2} \log \operatorname{det}\left(\alpha_{2}^{-1} \alpha_{1}\right) .
\end{array}
$$

We also have $\tau_{r, a}$ introduced in section 7.1 , and $\Xi, v_{a i}, v_{r i}, \mathfrak{b}_{a i}, \mathfrak{b}_{r i}$ are the same as those in section 2.1.

We will now use $\alpha_{r}$ to raise and lower $i, j$ indices. The time covariant derivative is the same as (2.14) except that one should use $E_{r}$ defined as in (D.4). and the spatial covariant derivatives are the same as (2.15)-(2.17) except that $\tilde{\Gamma}_{j k}^{i}$ should be replaced by that associated with $\alpha_{r i j}$, more explicitly,

$$
\tilde{\Gamma}_{j k}^{i} \equiv \frac{1}{2} \alpha_{r}^{i l}\left(d_{j} \alpha_{r k l}+d_{k} \alpha_{r j l}-d_{l} \alpha_{r j k}\right)=\Gamma_{j k}^{i}+\frac{1}{2} \alpha_{r}^{i l}\left(v_{r j} \partial_{0} \alpha_{r k l}+v_{r k} \partial_{0} \alpha_{r j l}-v_{r l} \partial_{0} \alpha_{r j k}\right)
$$

with $\Gamma_{j k}^{i}$ the Christoffel symbol corresponding to $\alpha_{r}$.

We can write the action as

$$
\int d^{d} \sigma \sqrt{\alpha_{r}} E_{r} \mathcal{L}
$$

where the integration measure $\sqrt{\alpha_{r}} E_{r}$ differs from that of (2.6) by some factors of $\tau$. Note that equation (7.5) implies that there cannot be any dependence on $\tau_{r}, \tau_{a}$ other than those included in $\hat{h}_{s a b} . \mathcal{L}^{(1)}$ to first derivative order and $\mathcal{L}^{(2)}$ to zeroth derivative order are given by (2.9)-(2.12) and (2.19) that one should eliminate all terms which involve $\tau$ explicitly and all coefficients are functions of $\hat{\mu}_{r}$ only.

We now write down $\mathcal{L}^{(1)}$ to second derivative order for neutral conformal fluids. For this purpose we need to use the the torsion $\mathfrak{t}_{i j}$ and various curvatures introduced in section $\mathrm{V}$ A3 
of [1] except that they should be accordingly modified as those associated with $\alpha_{r}$. The torsion $\mathfrak{t}_{i j}$ is defined by

$$
\left[D_{i}, D_{j}\right] \phi \equiv \mathfrak{t}_{i j} D_{0} \phi, \quad \mathfrak{t}_{i j}=E_{r}\left(d_{i} v_{r j}-d_{j} v_{r i}\right),
$$

and the "Riemann tensor" $\tilde{R}_{l i j}^{k}$ by

$$
\left[D_{i}, D_{j}\right] \phi_{k}=\tilde{R}_{i j k}^{l} \phi_{l}+\mathfrak{t}_{i j} D_{0} \phi_{k}
$$

with

$$
\tilde{R}_{i j k}^{l}=d_{j} \tilde{\Gamma}_{i k}^{l}-d_{i} \tilde{\Gamma}_{j k}^{l}+\tilde{\Gamma}_{k i}^{m} \tilde{\Gamma}_{j m}^{l}-\tilde{\Gamma}_{k j}^{m} \tilde{\Gamma}_{i m}^{l}
$$

Note that

$$
\tilde{R}_{i j k l}+\tilde{R}_{i j l k}=-\mathfrak{t}_{i j} D_{0} \alpha_{r k l} .
$$

and as a result, there are two "Ricci tensors":

$$
\tilde{R}_{i k}^{1}=\tilde{R}_{i j k}^{j}, \quad \tilde{R}_{i k}^{2}=\tilde{R}_{i j k}{ }^{j},
$$

neither of which is symmetric. We also introduce

$$
W_{i k}=\tilde{R}_{i k}^{1}+\tilde{R}_{i k}^{2}=-\mathfrak{t}_{i j} \alpha_{r}^{j l} D_{0} \alpha_{r k l}, \quad S_{i k}=\frac{1}{2}\left(\tilde{R}_{i k}^{1}-\tilde{R}_{i k}^{2}\right),
$$

where the second equality of the first equation follows from (D.11).

Now at second derivative order $\mathcal{L}^{(1)}$ can be written as

$$
\begin{aligned}
\mathcal{L}_{2}^{(1)}= & f_{1} E_{a}+f_{2} \chi_{a}+\lambda_{21} V_{a}^{i} \alpha^{j k} D_{j} D_{0} \alpha_{i k}+\lambda_{22} V_{a}^{i} \alpha^{j k} D_{j} \mathfrak{t}_{i k} \\
& -\Xi^{i j}\left[\frac{\tilde{\eta}}{2} D_{0} \alpha_{r i j}+\eta_{1} D_{0}^{2} \alpha_{i j}+\eta_{2} D_{0} \alpha_{i k} D_{0} \alpha_{j l} \alpha^{k l}+\eta_{3} S_{i j}+\eta_{4} \alpha^{k l} \mathfrak{t}_{k i} \mathfrak{t}_{j l}+\eta_{5} W_{i j}\right],
\end{aligned}
$$

where

$$
f_{1}=f_{11}+f_{15} \operatorname{tr}\left(D_{0} \alpha \alpha^{-1} D_{0} \alpha \alpha^{-1}\right)+f_{16} \operatorname{tr}\left(\alpha^{-1} D_{0}^{2} \alpha\right)+f_{17} S_{i j} \alpha_{r}^{i j}+f_{18} W_{i j} \alpha_{r}^{i j}+f_{19} \mathfrak{t}^{i j} \mathfrak{t}_{i j}
$$

and $f_{2}$ has the same structure as $f_{1}$. In (D.14)-(D.15), all coefficients are constants, and we have dropped terms which vanish on the zeroth order equations of motion:

$$
\operatorname{Tr}\left(\alpha_{r}^{-1} D_{0} \alpha_{r}\right)=0, \quad D_{i} E_{r}=0 \text {. }
$$

The $\hbar \rightarrow 0$ limit of the second line of (D.14) gives the second order stress tensor (7.24) with the correspondence

$$
\begin{aligned}
\eta_{1} & =-\frac{1}{8}\left(2 f_{5}+f_{1}\right), & \eta_{2} & =\frac{1}{32}\left(8 f_{5}-4 f_{2}+7 f_{1}\right), \\
\eta_{3} & =-\frac{1}{2} f_{1}, & \eta_{4} & =\frac{1}{32}\left(4 f_{3}+11 f_{1}\right), \quad \eta_{5}=-\frac{1}{8}\left(2 f_{5}+f_{4}+f_{1}\right) .
\end{aligned}
$$




\section{E Useful identities}

In this appendix we present the identities used in section 7. From

$$
\left[\hat{\nabla}_{\mu}, \hat{\nabla}_{\nu}\right] \beta_{\alpha}=\hat{R}_{\mu \nu \alpha}^{\beta} \beta_{\beta}
$$

and contracting with $\hat{\Delta}_{\mu \nu}$ or $\beta_{\mu}$, we find

$$
\begin{aligned}
\hat{R}_{\mu \nu} \beta^{\mu} \beta^{\nu} & =-\frac{1}{4}\left(\hat{\sigma}^{2}-\hat{\omega}^{2}\right) \\
\hat{\partial} \hat{\sigma}_{\mu \nu} & =2 \hat{R}_{\alpha \mu \nu \beta} \beta^{\alpha} \beta^{\beta}-\frac{1}{2}\left(\hat{\sigma}_{\mu}^{\alpha} \hat{\sigma}_{\alpha \nu}+\hat{\omega}_{\mu}^{\alpha} \hat{\omega}_{\alpha \nu}\right) \\
\hat{\partial} \hat{\omega}_{\mu \nu} & =\hat{\sigma}_{[\mu}{ }^{\alpha} \hat{\omega}_{\nu] \alpha} \\
\hat{\Delta}_{\alpha}^{\mu} \hat{\nabla}_{\mu} \hat{\nabla}_{\beta} \hat{\sigma}^{\alpha \beta} & =2 \hat{\Delta}^{\mu \alpha} \hat{\nabla}_{\mu}\left(\hat{R}_{\alpha \beta} \beta^{\beta}\right)-\frac{1}{2} \hat{\partial}\left(\hat{\omega}^{2}\right),
\end{aligned}
$$

where $\hat{R}_{\mu \nu}=\hat{R}_{\mu \alpha \nu}{ }^{\alpha}$, and where we used that $\hat{\nabla}_{\mu} \beta^{\mu}=\beta^{\mu} \hat{\nabla}_{\mu} \beta^{\alpha}=0$. We also have the Bianchi identity $\hat{\nabla}_{\mu}\left(\hat{R}^{\mu \nu}-\frac{1}{2} \hat{g}^{\mu \nu} \hat{R}\right)=0$.

Open Access. This article is distributed under the terms of the Creative Commons Attribution License (CC-BY 4.0), which permits any use, distribution and reproduction in any medium, provided the original author(s) and source are credited.

\section{References}

[1] M. Crossley, P. Glorioso and H. Liu, Effective field theory of dissipative fluids, arXiv: 1511.03646 [INSPIRE].

[2] S. Dubovsky, T. Gregoire, A. Nicolis and R. Rattazzi, Null energy condition and superluminal propagation, JHEP 03 (2006) 025 [hep-th/0512260] [INSPIRE].

[3] S. Dubovsky, L. Hui, A. Nicolis and D.T. Son, Effective field theory for hydrodynamics: thermodynamics and the derivative expansion, Phys. Rev. D 85 (2012) 085029 [arXiv: 1107.0731] [INSPIRE].

[4] S. Endlich, A. Nicolis, R.A. Porto and J. Wang, Dissipation in the effective field theory for hydrodynamics: First order effects, Phys. Rev. D 88 (2013) 105001 [arXiv:1211.6461] [INSPIRE].

[5] S. Dubovsky, L. Hui and A. Nicolis, Effective field theory for hydrodynamics: Wess-Zumino term and anomalies in two spacetime dimensions, Phys. Rev. D 89 (2014) 045016 [arXiv: 1107.0732] [INSPIRE].

[6] S. Endlich, A. Nicolis, R. Rattazzi and J. Wang, The Quantum mechanics of perfect fluids, JHEP 04 (2011) 102 [arXiv: 1011.6396] [INSPIRE].

[7] A. Nicolis and D.T. Son, Hall viscosity from effective field theory, arXiv:1103.2137 [INSPIRE].

[8] A. Nicolis, Low-energy effective field theory for finite-temperature relativistic superfluids, arXiv: 1108.2513 [INSPIRE]. 
[9] L.V. Delacrétaz, A. Nicolis, R. Penco and R.A. Rosen, Wess-Zumino Terms for Relativistic Fluids, Superfluids, Solids and Supersolids, Phys. Rev. Lett. 114 (2015) 091601 [arXiv: 1403.6509] [INSPIRE].

[10] M. Geracie and D.T. Son, Effective field theory for fluids: Hall viscosity from a Wess-Zumino-Witten term, JHEP 11 (2014) 004 [arXiv: 1402.1146] [INSPIRE].

[11] S. Grozdanov and J. Polonyi, Viscosity and dissipative hydrodynamics from effective field theory, Phys. Rev. D 91 (2015) 105031 [arXiv:1305.3670] [INSPIRE].

[12] M. Harder, P. Kovtun and A. Ritz, On thermal fluctuations and the generating functional in relativistic hydrodynamics, JHEP 07 (2015) 025 [arXiv: 1502.03076] [INSPIRE].

[13] P. Kovtun, G.D. Moore and P. Romatschke, Towards an effective action for relativistic dissipative hydrodynamics, JHEP 07 (2014) 123 [arXiv:1405.3967] [INSPIRE].

[14] F.M. Haehl, R. Loganayagam and M. Rangamani, The eightfold way to dissipation, Phys. Rev. Lett. 114 (2015) 201601 [arXiv:1412.1090] [INSPIRE].

[15] F.M. Haehl, R. Loganayagam and M. Rangamani, Adiabatic hydrodynamics: The eightfold way to dissipation, JHEP 05 (2015) 060 [arXiv: 1502.00636] [INSPIRE].

[16] F.M. Haehl, R. Loganayagam and M. Rangamani, The Fluid Manifesto: Emergent symmetries, hydrodynamics and black holes, JHEP 01 (2016) 184 [arXiv:1510.02494] [INSPIRE].

[17] F.M. Haehl, R. Loganayagam and M. Rangamani, Topological $\sigma$-models \& dissipative hydrodynamics, JHEP 04 (2016) 039 [arXiv: 1511.07809] [INSPIRE].

[18] F.M. Haehl, R. Loganayagam and M. Rangamani, Schwinger-Keldysh formalism. Part I: BRST symmetries and superspace, JHEP 06 (2017) 069 [arXiv:1610.01940] [INSPIRE].

[19] F.M. Haehl, R. Loganayagam and M. Rangamani, Schwinger-Keldysh formalism. Part II: thermal equivariant cohomology, JHEP 06 (2017) 070 [arXiv:1610.01941] [INSPIRE].

[20] N. Andersson and G.L. Comer, A covariant action principle for dissipative fluid dynamics: From formalism to fundamental physics, Class. Quant. Grav. 32 (2015) 075008 [arXiv: 1306.3345] [INSPIRE].

[21] S. Floerchinger, Variational principle for theories with dissipation from analytic continuation, JHEP 09 (2016) 099 [arXiv: 1603.07148] [INSPIRE].

[22] D. Nickel and D.T. Son, Deconstructing holographic liquids, New J. Phys. 13 (2011) 075010 [arXiv: 1009.3094] [INSPIRE].

[23] J. de Boer, M.P. Heller and N. Pinzani-Fokeeva, Effective actions for relativistic fluids from holography, JHEP 08 (2015) 086 [arXiv:1504.07616] [INSPIRE].

[24] M. Crossley, P. Glorioso, H. Liu and Y. Wang, Off-shell hydrodynamics from holography, JHEP 02 (2016) 124 [arXiv: 1504.07611] [INSPIRE].

[25] J. Zinn-Justin, Quantum Field Theory and Critical Phenomena, Clarendon Press, Oxford U.K. (2002).

[26] P. Gao and H. Liu, Emergent Supersymmetry in Local Equilibrium Systems, arXiv: 1701.07445 [INSPIRE].

[27] K. Jensen, N. Pinzani-Fokeeva and A. Yarom, Dissipative hydrodynamics in superspace, arXiv: 1701.07436 [INSPIRE]. 
[28] P. Glorioso and H. Liu, The second law of thermodynamics from symmetry and unitarity, arXiv: 1612.07705 [INSPIRE].

[29] N. Banerjee, J. Bhattacharya, S. Bhattacharyya, S. Jain, S. Minwalla and T. Sharma, Constraints on Fluid Dynamics from Equilibrium Partition Functions, JHEP 09 (2012) 046 [arXiv: 1203.3544] [INSPIRE].

[30] K. Jensen, M. Kaminski, P. Kovtun, R. Meyer, A. Ritz and A. Yarom, Towards hydrodynamics without an entropy current, Phys. Rev. Lett. 109 (2012) 101601 [arXiv: 1203.3556] [INSPIRE].

[31] M. Haack and A. Yarom, Universality of second order transport coefficients from the gauge-string duality, Nucl. Phys. B 813 (2009) 140 [arXiv:0811.1794] [InSPIRE].

[32] E. Shaverin and A. Yarom, Universality of second order transport in Gauss-Bonnet gravity, JHEP 04 (2013) 013 [arXiv:1211.1979] [InSPIRE].

[33] S. Grozdanov and A.O. Starinets, On the universal identity in second order hydrodynamics, JHEP 03 (2015) 007 [arXiv: 1412.5685] [INSPIRE].

[34] S. Grozdanov and A.O. Starinets, Zero-viscosity limit in a holographic Gauss-Bonnet liquid, Theor. Math. Phys. 182 (2015) 61 [Teor. Mat. Fiz. 182 (2014) 76].

[35] E. Shaverin, A breakdown of a universal hydrodynamic relation in Gauss-Bonnet gravity, arXiv: 1509.05418 [INSPIRE].

[36] P. Romatschke, Relativistic Viscous Fluid Dynamics and Non-Equilibrium Entropy, Class. Quant. Grav. 27 (2010) 025006 [arXiv:0906.4787] [INSPIRE].

[37] S. Bhattacharyya, Constraints on the second order transport coefficients of an uncharged fluid, JHEP 07 (2012) 104 [arXiv: 1201.4654] [INSPIRE].

[38] S. Bhattacharyya, Entropy Current from Partition Function: One Example, JHEP 07 (2014) 139 [arXiv: 1403.7639] [INSPIRE]. 